\title{
Application of advanced magnetic resonance imaging in glaucoma: a narrative review
}

\author{
Longdan Kang, Chao Wan^ \\ Department of Ophthalmology, the First Hospital of China Medical University, Shenyang, China
}

Contributions: (I) Conception and design: C Wan; (II) Administrative support: C Wan; (III) Provision of study materials or patients: Both authors; (IV) Collection and assembly of data: Both authors; (V) Data analysis and interpretation: L Kang; (VI) Manuscript writing: Both authors; (VII) Final approval of manuscript: Both authors.

Correspondence to: Chao Wan. 155 Nanjing North Street, Heping District, Shenyang 110001, China. Email: hundred2005@126.com.

\begin{abstract}
Glaucoma is a group of eye diseases characterized by progressive degeneration of the optic nerve head and retinal ganglion cells and corresponding visual field defects. In recent years, mounting evidence has shown that glaucoma-related damage may not be limited to the degeneration of retinal ganglion cells or the optic nerve head. The entire structure of the visual pathway may be degraded, and the degradation may even extend to some non-visual brain regions. We know that advanced morphological, functional, and metabolic magnetic resonance technologies provide a means to observe quantitatively and in real time the state of brain function. Advanced magnetic resonance imaging (MRI) techniques provide additional diagnostic markers for glaucoma, which are related to known potential histopathological changes. Many researchers in China and globally have conducted clinical and imaging studies on glaucoma. However, they are scattered, and we still need to systematically sort out the advanced MRI related to glaucoma. We reviewed literature published in any language and included all studies that were able to be translated into English from 1 January 1980 to 31 July 2021. Our literature search focused on emerging magnetic resonance neuroimaging techniques for the study of glaucoma. We then identified each functional area of the brain of glaucoma patients through the integration of anatomy, image, and function. The aim was to provide more information about the occurrence and development of glaucoma diseases. From the perspective of neuroimaging, our study provides a research basis to explain the possible mechanism of the occurrence and development of glaucoma. This knowledge gained from these techniques enables us to more clearly observe the damage glaucoma causes to the whole visual pathway. Our study provides new insights into glaucoma-induced changes to the brain. Our findings may enable the progress of these changes to be analyzed and inspire new neuroprotective therapeutic strategies for patients with glaucoma in the future.
\end{abstract}

Keywords: Glaucoma; magnetic resonance imaging (MRI); neurodegeneration; functional connection

Submitted Aug 08, 2021. Accepted for publication Nov 26, 2021.

doi: 10.21037/qims-21-790

View this article at: https://dx.doi.org/10.21037/qims-21-790

\footnotetext{
^ ORCID: 0000-0002-7414-0773.
} 


\section{Introduction}

Glaucoma is a group of eye diseases characterized by progressive degeneration of the optic nerve head $(\mathrm{ONH})$, loss of retinal ganglion cells (RGCs), and corresponding visual field (VF) defects (1). Glaucoma is the leading cause of irreversible blindness in the world. Currently, there are about 76 million glaucoma patients worldwide, which is expected to increase to 112 million by the year 2040. Every year, between 5.1 and 6.8 million people are affected by glaucoma and become blind, often in both eyes as glaucoma is usually binocular, which causes huge losses to society, families, and individuals (2). Glaucoma has been described as a silent vision thief because it lacks obvious visual deterioration and clinical symptoms during the early stage when the vision of glaucoma patients is being eroded (3). It is vitally important to intervene as soon as possible in the course of glaucoma before major and irreversible damage occurs (4). Therefore, more effective structural and functional measurements are needed for its early diagnosis.

Although some risk factors for glaucoma have been identified (namely high intraocular pressure (IOP), age, high myopia, race, and heredity), its pathophysiological mechanism is still unclear (5-7). In the human retina, glaucoma mainly leads to the selective death of RGCs. The dendrites of RGCs constitute the optic nerve, which provides most of the input to the primary visual cortex. Therefore, a reduction of the number of viable RGCs should lead to reduction of the number of optic nerve dendrites, which should result in the reduction of sensory inputs in the primary motor cortex and a corresponding reduction of the number of functional connection (FC) with the secondary visual cortex. That is, glaucoma-related damage may not be limited to degeneration of RGCs or the $\mathrm{ONH}$; the whole visual pathway may degenerate, including the optic nerve, lateral geniculate nucleus (LGN), and the visual cortex, and degeneration may even extend to some non-visual brain regions $(8,9)$. This raises the question of whether glaucoma should be considered a brain disease (10-12).

When evaluating glaucoma in vivo, structural analysis is usually limited to the parameters of the eye structure, such as those from macular-measured ganglion cell layer thickness, ONH, and retinal nerve fiber layer (RNFL) (13). Methods to evaluate and monitor glaucoma lesions in the central nervous system have become very important. Magnetic resonance imaging (MRI) has been widely used as a non-invasive imaging tool to evaluate intracranial structures. Traditional MRI is a highly sensitive diagnostic imaging method for evaluating internal structure, with special emphasis on soft tissue (14); however, such technology is usually not quantitative, direct comparability between scans is poor, and critical information for study of glaucoma is not captured in the brain examination results (15).

The advanced MRI is a series of emerging imaging techniques based on traditional MRI. In recent years, advanced MRI applications in glaucoma have gradually become a key research focus. The advanced MRI used in glaucoma brain detection mainly involves the following 7 types of methods: MRI quantitative morphometry; blood oxygenation level-dependent functional MRI (BOLDfMRI); diffusion-weighted imaging (DWI); magnetic resonance spectroscopy (MRS); diffusion tensor imaging (DTI); diffusion kurtosis imaging (DKI); and magnetization transfer imaging (MTI) (16-18). In this paper, we reviewed the application of these advanced MRI techniques in the study of glaucoma. The findings from our study may help researchers to understand the pathogenesis of glaucoma and improve access to vision health care services. We present the following article in accordance with the Narrative Review reporting checklist (available at https://dx.doi. org/10.21037/qims-21-790).

\section{Methods}

The purpose of this review was to synthesize existing literature on the emerging MRI neuroimaging techniques used for glaucoma when locating lesions in each brain functional area in the living brain of glaucoma patients by integrating structure, function, and metabolism.

Our research had a clear empirical basis and used qualitative, quantitative, and mixed methods research published in peer-reviewed journals and literature. All study designs were included. Electronic searches were conducted in the following electronic databases: PubMed, Google Scholar, and Web of Science. We searched literature published in any language and included all studies that could be translated into English from 1 January 1980 to 31 July 2021.

The eligibility criteria were developed to ensure that the included studies contained specific information needed to answer research questions about the emerging brain MRI neuroimaging techniques for glaucoma. For studies to be included in our research, they needed to meet the following criteria: (I) no language restrictions; and (II) the study focused on at least 1 emerging brain MRI neuroimaging technique for the study of glaucoma. Studies meeting 
the following criteria were excluded: (I) reporting drug and procedural interventions, considering that drug and procedural interventions themselves may have an impact on brain examination results; (II) glaucoma patients were not included as part of the study population; and (III) glaucoma was complicated with other diseases.

\section{Results}

\section{MRI quantitative morphometry}

In recent years, progress in neuroimaging technology has improved how accurately we can evaluate brain structure, volume, and microstructural damage. Quantitative morphological studies can assess changes in the volume and thickness of various specific structures in the brain of glaucoma patients. High resolution three-dimensional anatomical imaging is used for standardized morphometry to accurately and quantitatively evaluate the thickness and volume of the cerebral cortex. The techniques for quantitative evaluation of brain morphology can be divided into 2 types: voxel-based morphometry (VBM), and surface-based morphometry (SBM), The VBM technique uses a statistical method to allocate the probability that a voxel is occupied by gray matter, whereas SBM determines the vertices defining the interface surface of gray matter cerebrospinal fluid and gray and white matter, and uses these vertices to estimate the thickness of the cerebral cortex with submillimeter accuracy.

Using MRI VBM, Hernowo et al. found that the volume of all structures of the visual pathway in glaucoma patients was significantly reduced, including the optic nerve, optic chiasm, optic tract, LGN, and optic radiation (19). In the occipital lobe, MRI volume studies pay special attention to the visual and visual-related cortex. Recent reports using the volumetric MRI technique have observed a decrease in occipital surface area or visual cortex volume in both hemispheres of glaucoma patients (14).

Wang et al. performed structural MRI scanning on patients with high IOP glaucoma. Both SBM and VBM were used to evaluate the cortical thickness and subcortical gray matter volume of the participants. It was found that elevated IOP led to reduced cortical thickness in the bilateral superior temporal gyrus, bilateral superior parietal gyrus, bilateral lateral occipital gyrus, left fusiform gyrus, left medial orbitofrontal gyrus, right precentral gyrus, and the right superior frontal gyrus, and decreased gray matter volume of the right hippocampus, bilateral putamen, and bilateral thalamus (20).

In terms of the severity of glaucoma, Wang et al. used T1 weighted MRI images to conduct VBM and SBM analysis on the whole brain. They found that in patients with glaucoma, the left LGN volume was negatively correlated with the bilateral optic cup disk ratio, the right LGN volume was positively correlated with the average deviation of the right $\mathrm{VF}$, and the right $\mathrm{V} 1$ cortical thickness was negatively correlated with the right optic cup disk ratio. In patients with primary open angle glaucoma (POAG), these changes in brain visual structure can reflect the clinical severity of glaucoma (21).

\section{Blood oxygenation level dependent functional MRI}

Functional MRI (fMRI) can assess the function of the cortex in vivo based on the principle of cerebral metabolic changes caused by cortical activities (Figure 1). The BOLD effect is the most commonly used method to obtain information related to brain function. It is an indirect measurement of cortical activity. The BOLD-fMRI is a non-invasive imaging method that uses deoxyhemoglobin as the natural contrast agent in vivo to monitor blood oxygen levels of the brain in real-time. The changes in blood oxygen indirectly reflect the activities of local neurons. Both task-based fMRI (T-fMRI) and resting state fMRI (Rs-fMRI) are included in BOLD-fMRI: T-fMRI requires fine experimental design and related stimulation equipment, while Rs-fMRI does not. At present, the commonly used research indicators are regional homology $(\mathrm{ReHo})$, the amplitude of low frequency fluctuation (ALFF), and FC.

It is possible to use Rs-fMRI to study large-scale functional brain connections that depend on neural interactions over time. This technique allows synchronous and/or interregional dependent BOLD signal fluctuations to be performed by patients without any predetermined tasks. In the absence of visual stimuli, it is possible to study spontaneous brain connectivity in networks that support visual perception. Different Rs-fMRI studies of glaucoma have shown decreased connectivity in regions associated with vision. For example, a recent study by Minosse $e t$ al. using optical coherence tomography (OCT) found a significant correlation between Rs-fMRI and VF, and RNFL and the macular RGCs layer (25). Other studies have found changes of Rs-fMRI in some patients with ocular diseases, suggesting the potential neural mechanism of ocular diseases (26-30).

Studies have also shown that when the most vulnerable 


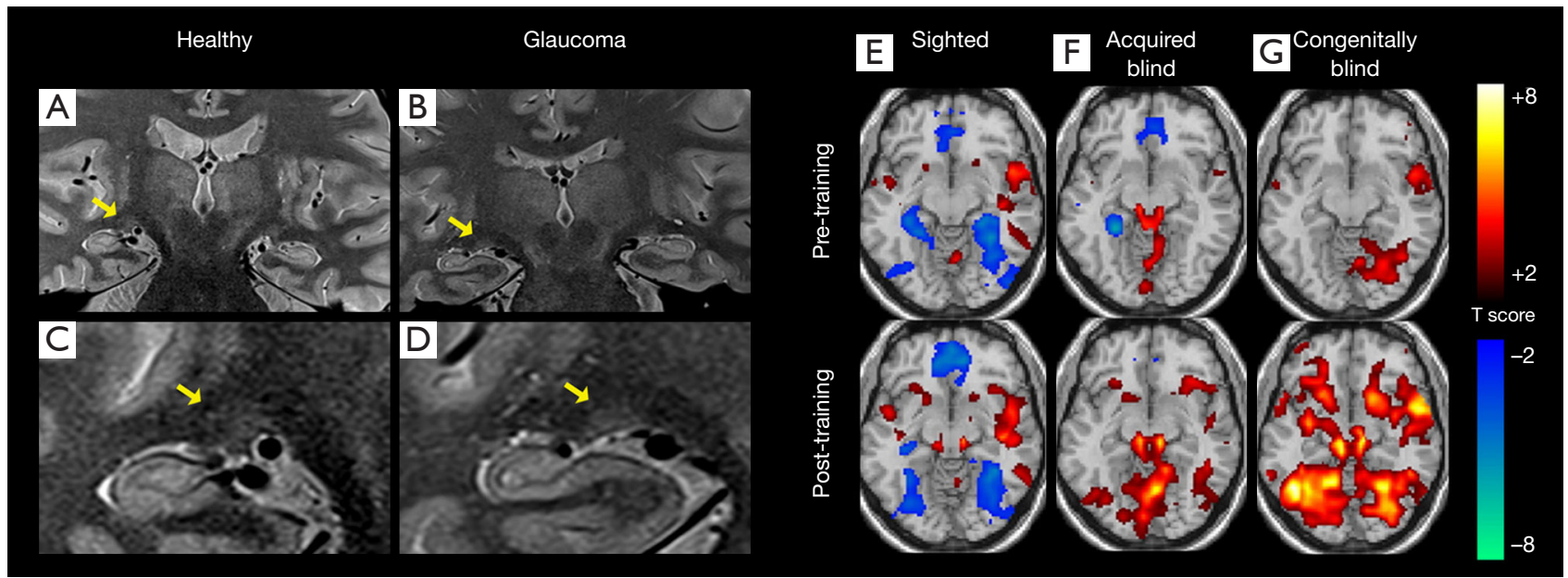

Figure 1 Structural and functional MRI of the visual brain nuclei. (B,D) Structural MRI indicating atrophy of the lateral geniculate nucleus in a glaucoma patient. (A,C) Structural MRI of a healthy subject for comparison. (E-G) Functional MRI demonstrating activation maps before (top row) and after (bottom row) task training for interpretation of cross-modal visual information inputted via auditory stimulation in three study cohorts: (E) sighted individuals, (F) acquired blind individuals, and (G) congenitally blind individuals. As compared to sighted individuals, those with acquired or congenital blindness demonstrated increased activity in the subcortical nuclei, suggesting that crossmodal sensory input is processed at this level in a distinct manner in this population. Reprinted with permission from Furlanetto et al. (22), Murphy et al. (23), and Sims et al. (24). MRI, magnetic resonance imaging.

area of $\mathrm{VF}$ in glaucoma is stimulated, the BOLD activity decreases in the corresponding area of the visual cortex. This decrease may be due to the loss of RGCs and the reduction of stimulation of the whole visual pathway, which reflects the reduction of afferent input from the eye. The decrease of BOLD activity seems to be more obvious in the primary visual cortex than in other higher visual regions (31).

Dai et al. used Rs-fMRI to analyze the FC of the visual cortex in patients with POAG and found changes in the $\mathrm{FC}$ of the visual cortex in POAG patients. The changes included those in the connection between the visual cortex and associated visual areas, as well as interruption of the connection between primary and advanced visual areas (32). In their study using Rs-fMRI, Li et al. found a change in the low-frequency fluctuation amplitude in patients with POAG. They proposed that POAG is a neurodegenerative disease involving multiple brain regions, including the visual cortex, default mode network (DMN), limbic system, and the motor and sensory networks (33). Furthermore, Li et al. used Rs-fMRI fractional ALFF technology to study the changes of spontaneous brain activity in normal tension glaucoma (NTG) patients. They found that abnormal spontaneous activity was detected in multiple brain regions of NTG patients, which was significantly negatively correlated with the changes of RNFL thickness detected by
OCT (34). The activity changes of these brain regions can be used as effective clinical indicators of glaucoma.

In addition, Qing et al. found that POAG results in decreased cortical activity in the visual cortex, including the central region (35). Primary angle closure glaucoma (PACG) showed decreased activity in the bilateral secondary visual cortex (BA18). In patients with POAG, FCs decreased between the visual cortex and the right inferior temporal gyrus, the left fusiform gyrus, the left middle occipital gyrus, the right superior occipital gyrus, the left central posterior gyrus, the right central anterior gyrus, and the left anterior cerebellar lobe, while patients with PACG showed an abnormal average of the bilateral middle occipital gyrus $\beta$ value. Patients with POAG had a significant decrease in the unilateral anisotropy fraction in the left hemisphere of the caudate nucleus, and the fibers of the left hemisphere in caudate nucleus reached the inferior parietal lobe, the dorsal parietal lobe, and the occipital lobe. Patients with PACG showed had decreased ReHo values in the left cClaustrum lobe and the right paracentral lobe (BA4) (36). Wang et al. used Rs-fMRI to study changes of spontaneous neuronal activity and FC mode in patients with PACG. They found that patients with PACG had abnormal spontaneous neural activity and connections in several brain regions that mainly related to vision and vision-related functions. These 
findings suggest that the fractional ALFF values of the left cuneiform bone and the bilateral superior frontal gyrus may be supplementary biomarkers to evaluate the severity of the disease (37).

In addition, when studying the FC of glaucoma patients, existing research found that the reduction of BOLD activity may also involve many areas of non-visual pathways. Huang et al. used fMRI to evaluate the relationship between the local characteristics of spontaneous brain activity and the thickness of RNFL in PACG patients. They found that PACG was mainly related to frontal lobe dysfunction (38). Chen et al. used the ReHo method and found that PACG was involved in abnormal spontaneous brain activities in multiple brain regions, such as the left fusiform gyrus, left anterior cerebellar lobe, right frontotemporal space and right insula, bilateral middle occipital gyrus, and the right paracentral lobule (36). Jiang et al. studied frequencydependent spontaneous neural activity of patients with PACG and found that they showed abnormal spontaneous neural activity in the visual cortex, sensorimotor cortex, frontal lobe, frontal parietal lobe network, and the DMN. This indicated that the visual, cognitive, and emotional functions of individuals with PACG may be impaired (39). These abnormal spontaneous neural activities shown by the clinical manifestations may suggest the potential pathological mechanism of PACG.

In addition to primary glaucoma, abnormalities can also occur in brain regions of patients with neovascular glaucoma (NVG). Zhang et al. used Rs-fMRI to analyze the brains of patients with NVG and revealed dysfunction in the brain regions of the right romantic operculum, left antagonist cingulate and paracingulate gyri, left precuneus, and the right caudate (40). Peng et al. observed abnormal spontaneous activity in NVG patients in the right cuneus, right middle occipital gyrus, left cingulate gyrus, right precuneus, left medical frontal gyrus, right superior frontal gyrus, and left middle frontal gyrus. These abnormal changes in specific brain regions can be considered possible clinical indicators of NVG (41). Wu et al. found that NVG involves abnormal spontaneous FCs of the primary visual cortex (V1) in different brain regions, including regions related to visual and cognitive functions (42). These findings may help to reveal the potential neural mechanisms of brain activity associated with NVG.

In terms of the severity of glaucoma, some researchers have observed that in mild and moderate cases of glaucoma, the primary visual cortex seems to be more affected than other advanced visual areas. For example, abnormal spontaneous neural activity in the left wedge, bilateral middle temporal gyrus, and the right prefrontal cortex have been shown to be associated with the severity of POAG (33).

In addition, Wang et al. also found that glaucoma patients had hemispheric synchronous injury. In a study of glaucoma patients using Rs-fMRI, they found that the homologous connection between hemispheres in BA17/ BA18/BA19 was reduced. This confirmed that there may be changes in isopositional FC and anatomic connections between hemispheres in POAG patients (43).

At present, fMRI is a very interesting clinical research tool for understanding how glaucoma functionally affects the central nervous system; however, it still has some limitations. First, fMRI technology is based on the premise that neural networks are associated with anatomic known visual regions, but other neural network components which may be affected by glaucoma cannot be clearly defined. Second, fMRI signal has low-temporal resolution due to down-sampling and indirectly measures underlying neuronal activity.

\section{$D W I$}

The DWI method is a non-invasive functional imaging way of analyzing the internal structure and tissue components of lesions at the molecular motion level by detecting the diffusion movement of water molecules in vivo. In neuroradiology, DWI is highly sensitive and can enable early identification of ischemic tissue injury and inflammatory diseases (44). It can be used to quantify the structural integrity of axons, which is useful for longitudinal monitoring of the progression of neuropathy. Haykal et al. used a new framework for DWI analysis, fixed-based analysis, to describe the neurodegeneration of glaucomatous optic nerve from the changes of axon density and morphology. They found that compared with the control group, the optic nerve of glaucoma patients showed lower axial density and fiber bundle cross section (an estimate of morphological changes), indicating optic nerve axon loss and severe atrophy (Figure 2) (45). DWI can also be used to quantitatively evaluate lesions by measuring the apparent diffusion coefficient (ADC), mean diffusion (MD) coefficient, and fractional anisotropy (FA). The ADC is used to measure the amount of signal strength loss, while MD is the amount of average molecular motion and FA is the amount of direction dependence of ADC.

Early DWI studies used single shot echo planar imaging (ss-EPI), but the limited resolution of this technology led 

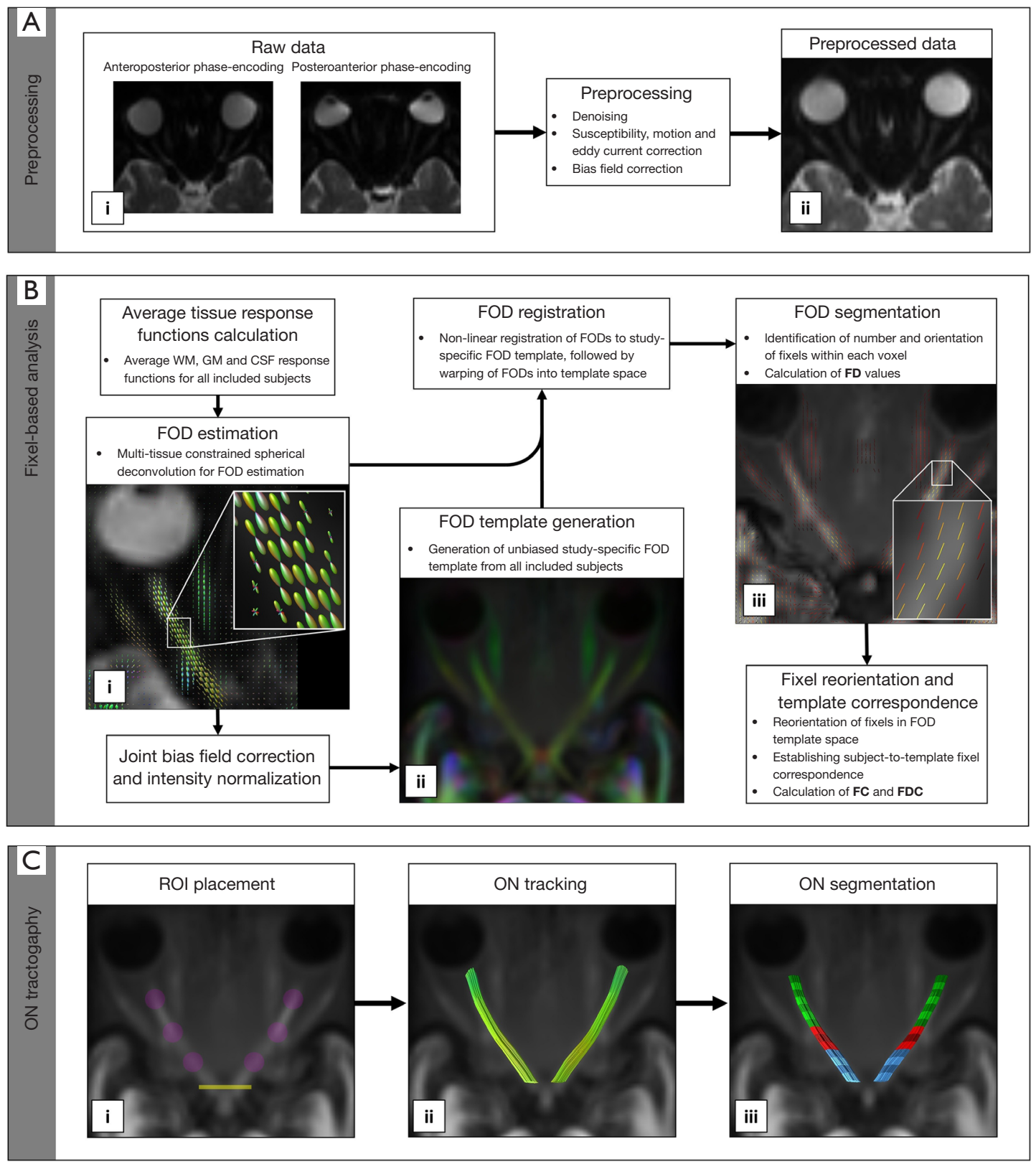

Figure 2 Overview of the data analysis pipeline. (A) Preprocessing of raw DWI data; i) example of single subject DWI scans acquired using anteroposterior and posteroanterior phase-encoding directions; ii) preprocessed DWI scan of the same subject. (B) Fixel-based analysis; i) a close-up view of FOD estimated for a single participant. The zoomed inset shows FODs in a segment of the ON; ii) a cropped image of the produced population template, color-coded based on the directionality of diffusion; iii) a cropped image of the identified fixels in a single participant. Fixels are color-coded based on their FD values, with yellow representing higher FD and red representing lower FD. The zoomed inset shows fixels in a segment of the ON. C) ON tracking and segmentation; i) placement of ROIs, where yellow shows the optic chiasm ROI, and magenta shows the visual representation of spherical ROIs placed along the ONs; ii) the result of deterministic tracking between the ROIs, color-coded by directionality; iii) ON segmentation. Each optic nerve is segmented into 14 sub-segments, and each sub-segment is assigned to an anatomical ON segment. Alternating shades of the same color represent different sub-segments of the same anatomical segment, where green is the intraorbital segment, red is the intracanalicular segment, and blue is the intracranial segment. Reprinted with permission from Haykal et al. (45). DWI, diffusion-weighted imaging; FOD, fiber orientation distribution; ON, optic nerve; ROIs, regions of interest; FD, fiber density. 
to problems such as incorrect volume measurement of the optic nerve, artifacts caused by cerebrospinal fluid and orbital fat, susceptibility changes of the tissue interface, and motion artifacts (46). Reading segment EPI (rs-EPI) is combined with parallel imaging to overcome these problems because doing so generates high-resolution DWI and significantly reduces the amount of sensitivity artifacts (47). This method provides robust correction for motion-induced phase artifacts and produces images within a clinically acceptable acquisition time. In the study of optic neuropathy, DWI was used for imaging in patients with acute visual loss to evaluate optic nerve axon injury. Another improved DWI method is called zoom EPI DWI, which uses focused or spatially selective excitation parallel transmission to reduce artifacts and improve image quality. This method produces a high-resolution image and reduces the scanning time, because it uses a reduced field of view in the phase coding direction, which results in an enlarged image. This technique is also known as reduced field of view EPI (rFOV-EPI) $(47,48)$.

Boye et al. used DWI to calculate the flow range ratio between intracranial cavity and the subarachnoid space of the optic nerve. They demonstrated a method for evaluating cerebrospinal fluid flow in the subarachnoid space of the optic nerve in a non-invasive manner, and found that the flow range ratio of NTG patients was significantly lower than that of healthy control participants (49). This finding suggests that impaired cerebrospinal fluid dynamics may play a role in the pathophysiology of NTG.

Cio et al. evaluated the global reorganization of the brain in POAG patients by combining DWI, multi-shell multitissue probabilistic tractography, graph theoretical measures, and a newly designed disruption index. They found that there were global differences in structural connectivity and local graph theoretical measures in glaucoma patients, and these changes far exceeded the main visual pathways. The results support the hypothesis that whole brain structural reorganization occurs in patients with glaucoma, which is specific to structural connections. This finding means that glaucoma can be categorized in the recently defined category of a brain disconnection syndrome (50).

Some researchers have used DWI evaluation to show the whole brain structural reorganization in POAG patients. The relationship between the measurement of whole brain structural connectivity and visual acuity index has also been reported. Di et al. found that the differences in global and local structural connectivity between POAG patients and the control group far exceeded the main visual pathways and were in the left talar gyrus, left occipital cortex, and the right lingual gyrus. There was a negative correlation between RNFL thickness and local measurement of the right amygdala, right inferior temporal gyrus, and the right temporal pole. The whole-brain structural reorganization in patients with POAG spans a variety of brain regions involved in visual processing, motor control, and emotional/ cognitive function. Patterns of brain structural changes associated with the clinical severity of POAG were also identified. Therefore, a hypothesis was proposed that the visual impairment of POAG can be driven by a combination of local (i.e., eye) and broader (i.e., brain) effects (51).

Hanekamp et al. processed DWI data using automated pipelines implemented by replicable web services to compare the white matter changes of glaucoma and nonglaucoma monocular patients. They found significant differences and that loss of visual input in monocular blindness may promote white matter reorganization outside the early visual system, but this reorganization may be reduced or is absent in glaucoma patients. The results provide indirect evidence that unknown factors may limit the reorganization of white matter after visual loss in glaucoma patients (52).

There are several limitations to DWI. First, DWI delineates that random Brownian motion of water in normal and pathological neural tissue environments changes. Reduced water diffusion has been shown to be sensitive to many diseases in the brain but is not specific to glaucoma. Therefore, the detection results of DWI may be affected by other factors and cannot be used as a biomarker of glaucoma neurodegeneration. Second, according to the partial volume effect and image slice direction, the diffusion limitation of the optic nerve may be ignored due to the small size of the optic nerve. Therefore, in the case of insufficient spatial sampling, the incidence of limited diffusion in the optic nerve may be underestimated. Thin slices (e.g., $3 \mathrm{~mm}$ ) arranged parallel to the optic nerve should improve the sensitivity of optic nerve diffusion limitation. Third, although the sensitivity and accuracy of rs-EPI and rFOVEPI are similar, their image quality in the orbital segment is significantly higher, and there are still artifacts in the optic canal segment and the intracranial segment.

\section{$D T I$}

Based on conventional DWI, the new neuroimaging technology of DTI is an MRI method that goes by the assumption that water molecules diffuse with a Gaussian 

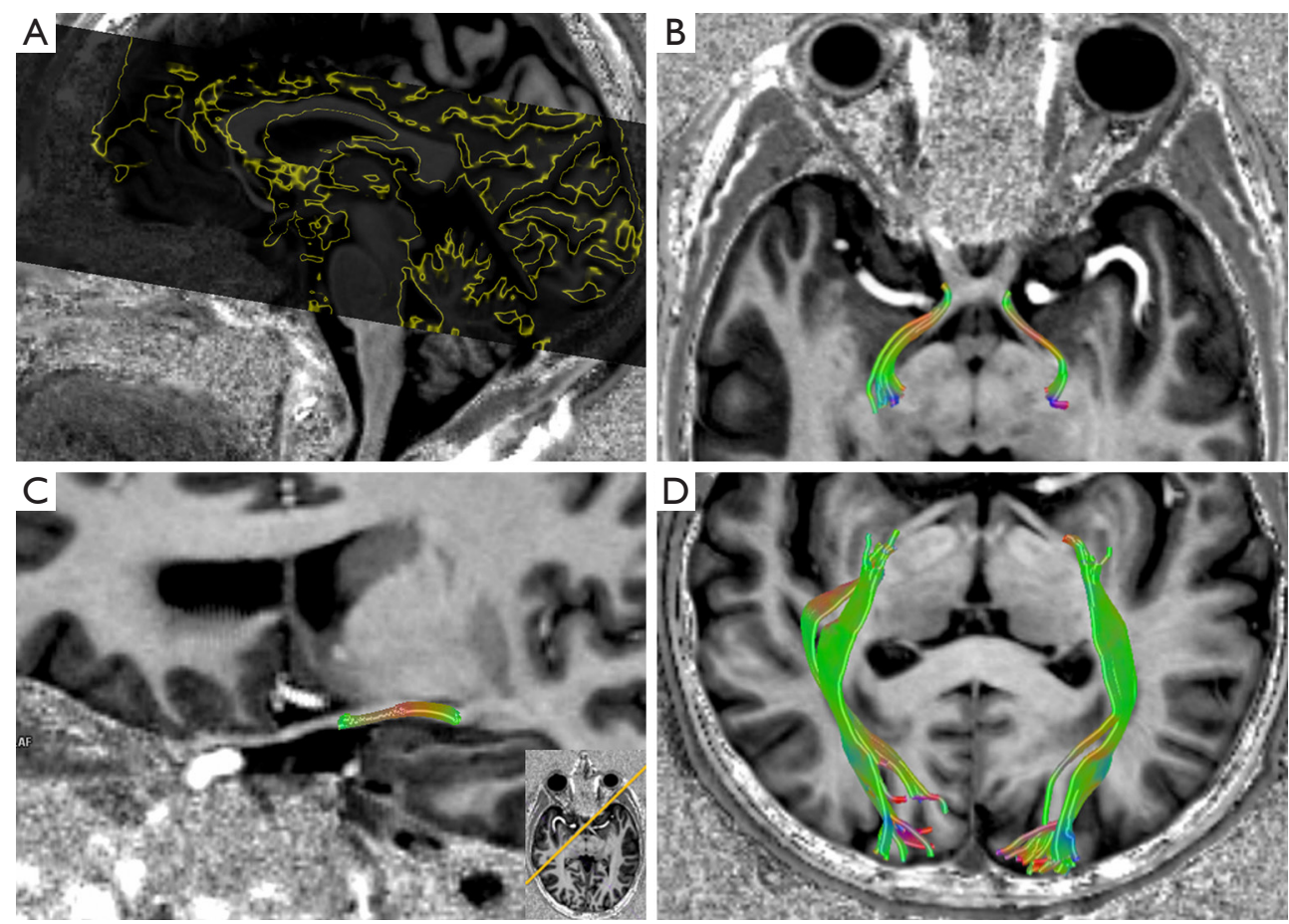

Figure 3 DTI. (A) DTI coverage of the visual pathway. (B and C) Reconstruction of the DTI dataset as selective tractography of the optic tract. (D) The optic radiation in normal tension glaucoma. Reprinted with permission from Schmidt et al. (53). DTI, diffusion tensor imaging.

distribution to measure and obtain the anisotropy information of different tissues (Figure 3). The dislocation of water molecules in nerve fibers and axons can be detected by the diffusion of water molecules along axons without a gadolinium contrast agent. Doing so can reveal abnormalities of the white matter structure and brain connection (54). The DTI technology is the most commonly used method in MRI, and it can quantitatively measure the integrity of microstructure and tissue in vivo. It has been widely used to study the differences of white matter bundles in the visual pathway in glaucoma patients. Microstructural differences of white matter structures have been found in the optic nerve, optic tract, optic chiasm, optic radiation, and the occipital lobe of glaucoma patients. The parameters of DTI include $\mathrm{FA}$, which indicates the directivity of the diffused signal, $M D$, axial diffusion (AD), and radial diffusion (RD), which represent the size and fraction of the diffusion signal. They are all measures of white matter damage. The decrease of $\mathrm{FA}$ and the increase of $\mathrm{MD}, \mathrm{AD}$, and $\mathrm{RD}$ are signs of axonal injury. The FA is considered a sensitive parameter for the diagnosis of glaucoma in the optic nerve, the optic tract, and optic radiation, and it is related to disease severity and age (55). Therefore, DTI provides information about nerve fibers and their pathways and estimates the integrity of nerve fibers by indirectly measuring the number of axons $(56,57)$. Based on this, it is believed that DTI may reveal early axonal injury with more sensitivity than a conventional MRI can.

Some researchers argue that DTI can be used to distinguish different glaucoma subtypes. Engelhorn et al. detected intracranial changes related to glaucoma and distinguished different glaucoma subtypes even in the early stage of the disease. They did this by using the calculation of DTI parameters of the visual pathway and the accompanying voxel-based method (58). In addition, compared with established ophthalmic examinations, the use of these new imaging techniques seems to enable earlier diagnosis of NTG r. El-Rafei et al. proposed a system based on DTI analysis of visual pathway fibers in optic radiation to detect and distinguish different types of glaucoma. They did this by using the support vector machine classifier to sort the features. Using visual pathway analysis, rather than traditional eye analysis, they demonstrated that DTI derived measures can distinguish POAG patients from healthy controls, NTG patients from healthy controls, and different 
types of glaucoma (POAG and NTG), with an accuracy of $92.4 \%, 100 \%$, and $98.3 \%$, respectively (59).

Similarly, the results of Schreiber et al. further support the hypothesis that glaucoma can be considered a neurodegenerative disease. Moreover, studies believes that the brain injury mechanism outside the visual pathway in POAG patients is not the direct diffusion of visual pathway degeneration, but the primary neuropathological process of the transmission mechanism of neurodegenerative lesions (60). Giorgio et al. found that compared with the healthy control group, patients with NTG and POAG had significantly more gray matter atrophy in their visual system and non-visual brain areas, and significantly more changes in DTI-derived anatomical connections. The POAG type has more obvious atrophy of visual cortex and higher axial diffusion of non-visual area. The study of NTG found that even if the IOP did not increase significantly, the whole visual system of the brain changed. This showed that the diffuse structural and functional abnormalities of the brain of a patient with glaucoma may, at least in part, be unrelated to the increase of IOP and subsequent retinal degeneration (61). Using DTI in the NTG population, Boucard et al. found that glaucoma was related to a low FA of visual radiation, forceps major, and corpus callosum, suggesting evidence of white matter degeneration in these sites. In particular, degeneration of the corpus callosum indicates that the existence of cerebral nerve degeneration goes beyond the explanation based on transmitted retinal and anterior lateral geniculate body (LGB) injury. Brain components independent of eye injury play a role in the etiology of glaucoma (60). In addition, Murai et al. found by DTI that there was a significant correlation between optic radiation axon injury and decreased cerebral glucose metabolism in the striate cortex of POAG patients (62).

Similar results were found in animal experiments. Takahashi et al. applied primate research results to show that DTI combined with spectral domain OCT can be used to track the early progress of optic neuropathy over time in the non-human primate high IOP model (63).

The DTI method has been widely used to evaluate microstructural abnormalities of white matter in the brain; however, it has some limitations. For example, due to the existence of organelles, cell membranes, and other barriers, water molecules often show non-Gaussian diffusion in biological tissues. Therefore, the practicability and sensitivity of the DTI model may not be completely optimal. In addition, DTI studies have shown damage to the integrity of the overall microstructure in the visual pathway of patients with glaucoma (64). In terms of the directional diffusion coefficient, some studies have attributed the decrease of FA to the increase of RD and the decrease or no change of $\mathrm{AD}$, suggesting that there is demyelination and axonal damage in the visual pathway of patients with glaucoma (65). However, studies showed that the decrease of FA in patients with glaucoma is driven by a greater increase of $\mathrm{RD}$ relative to $\mathrm{AD}$. Although $\mathrm{AD}$ and $\mathrm{RD}$ are sensitive indicators, they are not specific biomarkers of axon and myelin integrity (66). Therefore, the mechanism behind the difference of directional diffusion rate in glaucoma DTI research is not clear, and the pathophysiology of glaucoma may be more complex than the deterioration of the axon and myelin sheath.

\section{$D K I$}

The DKI method is a natural extension of the DTI model (Figure 4). Water diffusion in vivo moves in intercellular space and cells, and it does not have free motion. Therefore, the motion displacement of diffusion of real water molecules follows a non-Gaussian distribution (68). The greater that the diffusion of water molecules is limited by the surrounding environment, the more mixed the tissue components are in voxels, and the more significant the nonGaussian nature of diffusion. In order to quantify the degree to which diffusion deviates from the Gaussian distribution, Jensen et al. proposed the DKI model in 2005, which can be used to quantify the integrity of microstructure and tissue complexity even in the presence of cross fibers (69). The DKI scanning can be used to not only obtain diffusion indexes, such as DTI parameters, but also be used to obtain kurtosis parameters, such as mean kurtosis (MK), axial kurtosis (AK), and radial kurtosis (RK). The RK reflects the complexity of fiber orientation and vertical orientation $(70,71)$.

A key assumption of DTI is free water diffusion, or Gaussian diffusivity, which is characterized by the single exponential attenuation of the DWI signal on diffusivity sensitization (b value). Compared with traditional DTI, the image acquisition of DKI involves a higher $b$ value $\left(2,000 \mathrm{~s} / \mathrm{mm}^{2}\right)$ and a larger gradient direction. By experimenting with a high $b$ value, we can see the nonGaussian diffusion mode of gray matter and white matter, which may be due to the structural complexity of the cellular environment. The single exponential signal attenuation deviation observed at a high $\mathrm{b}$ value can be explained by a more detailed model, namely the DKI (72). The DKI 

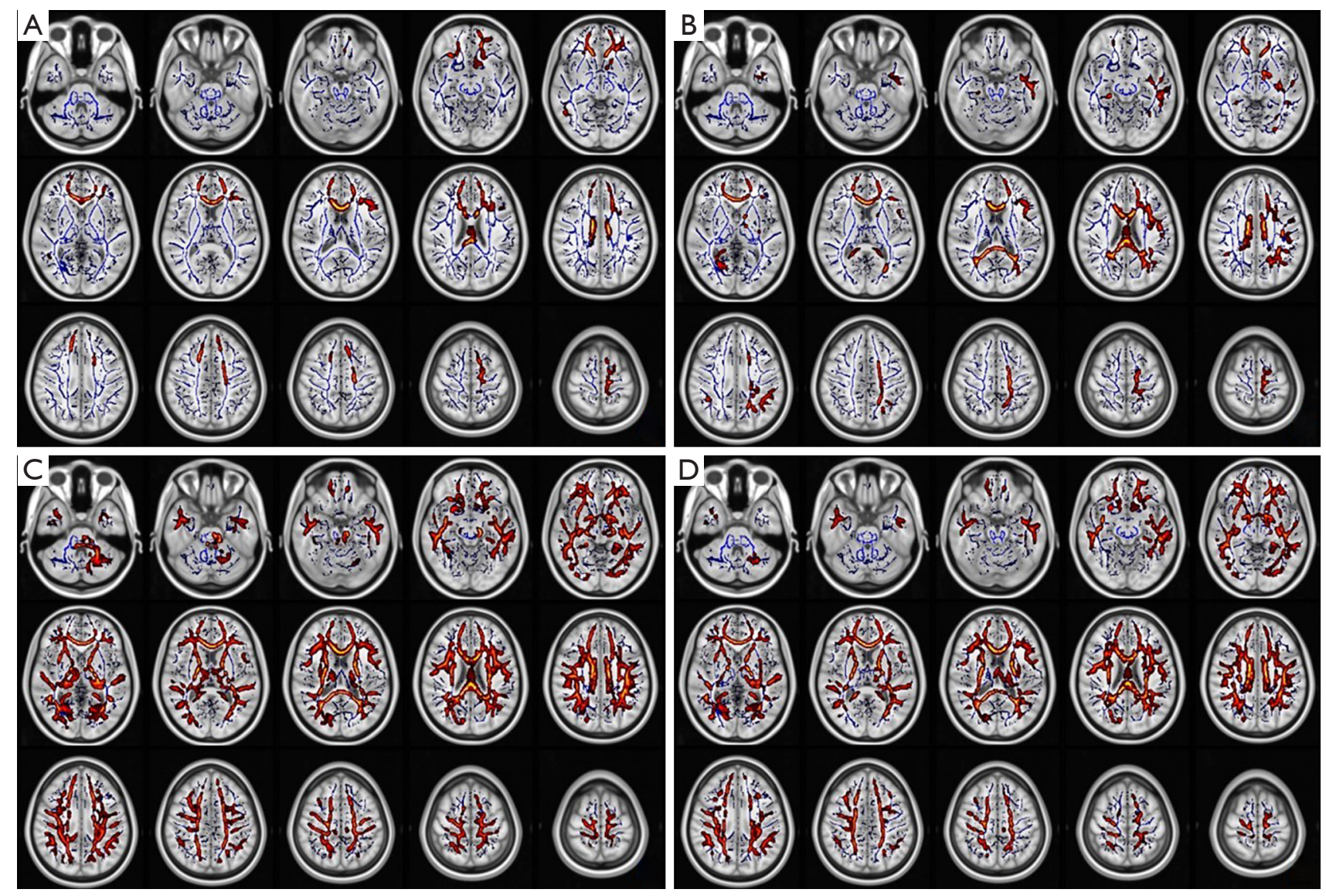

Figure 4 DKI. (A) Regions which displayed significant differences in FA (displayed as red and yellow). (B) Regions which displayed significant differences in KA (displayed as red and yellow). (C) Regions which displayed significant differences in MK (displayed as red and yellow). (D) Regions which displayed significant differences in RK (displayed as red and yellow). The white matter skeleton is shown in blue. Reprinted with permission from Nucci et al. (67). DKI, diffusion kurtosis imaging; FA, fractional anisotropy; KA, kurtosis anisotropy; MK, mean kurtosis; RK, radial kurtosis.

model shows higher sensitivity and specificity in evaluating neural tissue development and pathological changes, it helps to quantify specific neurodegenerative events in vivo, and it can provide more accurate and specific parameters for characterizing the microstructure environment of the brain. The DKI model is a useful biomarker of human brain tissue that is affected by the disease; therefore, it can better display the brain changes of patients with glaucoma (73).

MK from DKI is a better biomarker than FA and MD in detecting microstructure damage (68). Studies using DKI have shown that MK decreases along the brain visual pathway in patients with glaucoma. Xu et al. used 3T DKI to evaluate the damage to the microstructure of the visual pathway in patients with primary glaucoma. They found that glaucoma is a complex nervous system disease that affects the whole visual pathway. The RK of optic tract in glaucoma patients was significantly lower than that in healthy controls. This indicates that water diffusion perpendicular to the axon axis is less limited, which is consistent with the high RD in DTI (74).

Using DKI, Sun et al. found that in addition to the damage of axon integrity, glial cells were involved in glaucoma. In addition, through OCT and VF tests, DKI parameters were significantly correlated with clinical ophthalmic measurements, but not with DTI parameters. This showed that DKI provides a sensitive and comprehensive imaging biomarker to quantify the clinical severity of glaucoma damage in the white matter bundle, which is complementary to DTI (66).

The use of DKI has also been found useful in brain 

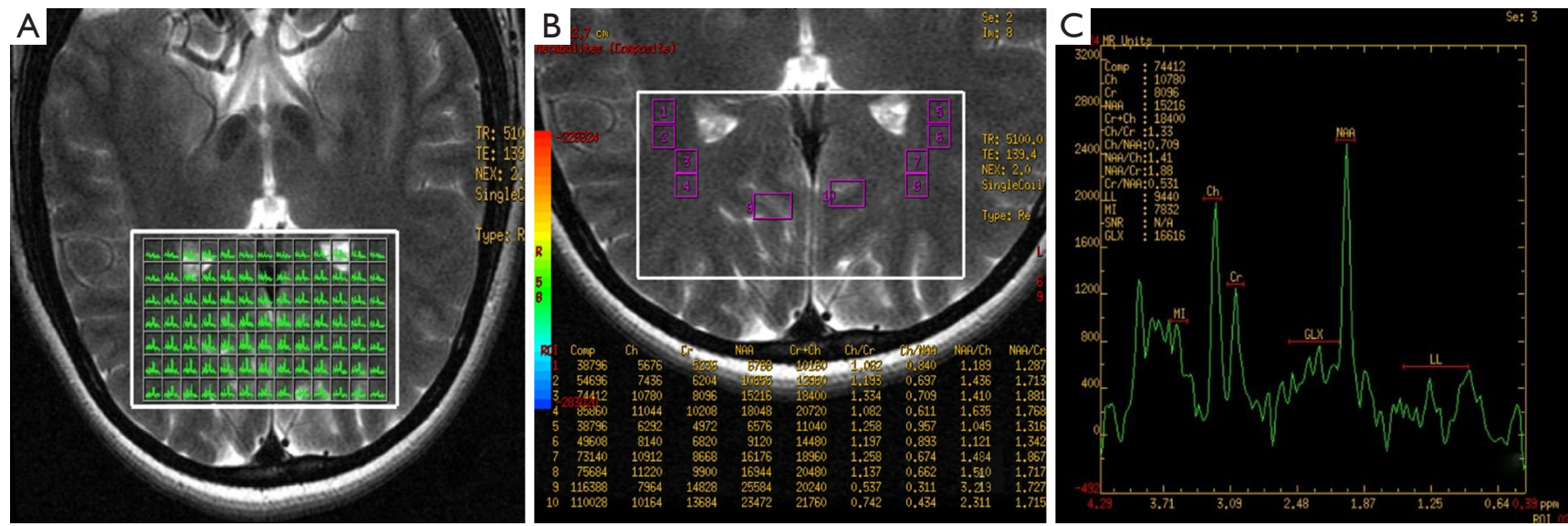

Figure 5 All the fixed-voxels in the VOI (white box). The size of each voxel was $31.6 \mathrm{~mm} 2$, as recommended by the software. (B) was the amplification of (A). We selected several individual voxels as the ROI in the bilateral GCT and the striate area. ROI 1 to ROI 8 , all of which were individual voxels, were distributed symmetrically in bilateral GCT. ROI 9 and ROI 10 were located in the striate area, and each contained six voxels. (C) the spectra of ROI 2 in the right GCT, with NAA and choline (Cho) peaks. Reprinted with permission from Zhang et al. (76). VOI, volume of interest; ROI, region of interest; GCT, geniculocalcarine tract; NAA, acetylaspartic acid.

regions outside the visual pathway. Nucci et al. used DKI to assess the difference between healthy controls and a mixed population of patients with POAG from stage I to $\mathrm{V}$ according to Hodapp-Parrish-Anderson VF injury classification. They found that there were wide differences in several indexes (MK, kurtosis anisotropy, RK, and FA) of white matter bundles between glaucoma patients and the control group. These indicators go far beyond the localization of the visual pathway and involve cognition, movement, facial recognition, and orientation functions, among others (67). Li et al. used DKI to study the information flow changes and microstructure abnormalities of the visual cortex in NTG. They showed that NTG leads to the reorganization of information flow between the visual cortex and other brain regions, which is consistent with damage to the brain's microstructure (75). These findings further support involvement of the brain in glaucoma and provide an explanation for many multi-domain injuries often observed in glaucoma patients.

\section{MRS}

The MRS is a non-invasive technology, which can detect, identify, and quantify biochemical compounds or metabolites in brain tissue (Figure 5). Due to the chemical heterogeneity of the human brain, there are various concentrations of important metabolites in normal brain structural areas, which provide different physiological and chemical information. Metabolites that can be measured using MRS include n-acetylaspartic acid (NAA), choline (Cho), creatine (Cr), lactate, glutamine, glutamate, lipids, and macromolecules. Barbosa Breda et al. accurately described the function of each metabolite and the peak position of the corresponding metabolite in the spectrum. They found that molecular biomarkers can help diagnose glaucoma and may lead to new targeted therapy (77).

Only a few studies have examined the changes of metabolite concentration in a visual pathway related to glaucoma, and these studies have involved different research sites. For example, in ocular tissue, Doganay et al. studied LGB and vitreous monomer proton MRS (1H-MRS) in 29 glaucoma patients and 13 healthy controls and found that lactate peaked in the vitreous of glaucoma patients. The glutamate-glutamine $(\mathrm{Glx}) / \mathrm{Cr}$ ratio in vitreous and LGB decreased significantly, but there were no changes in NAA/ $\mathrm{Cr}$ and Cho/Cr ratios in LGB (78).

Aksoy et al. investigated any metabolic changes of MRS in the whole brain's visual pathway in glaucoma patients and control groups and found that the values of NAA and Cho in the LGB of glaucoma patients decreased. In glaucoma cases, the NAA value of the visual cortex was negatively correlated with the cup plate ratio (79). Therefore, the use of MRS can reveal neurodegeneration of the LGB and the visual cortex in patients with glaucoma. These findings suggest that mapping the metabolic changes of the whole visual pathway by MRS will guide the treatment and follow- 
up plans of glaucoma patients.

In contrast, Sidek et al. compared the concentrations of metabolites in visual radiation between glaucoma patients and healthy controls using $1 \mathrm{H}-\mathrm{MRS}$. From this study, the ratio of metabolite concentration to $\mathrm{Cr}$ more reliably represented the change of visual radiation. They concluded that a progressive VF defect does not affect the absolute metabolite concentration in visual radiation. They also found that although there was clinical evidence of severe glaucoma, there was no significant metabolite change in visual radiation, which is related to glaucoma and its severity. However, the authors explained that this result may be due to the slowly progressive nature of the disease, because the large reduction of neuronal marker NAA occurs in the acute phase of cell degeneration. Another possible explanation is that the affected area may be too small to accurately measure, and more advanced MRS methods are needed (80). Later, a study conducted by Zhang et al. used multivoxel MRS to compare glaucoma patients with age and gender-matched healthy controls. In particular, polyvoxel $1 \mathrm{H}-\mathrm{MRS}$ was used to find changes of metabolite concentrations in the geniculocalcarine trace area and striate area. Significant reductions in $\mathrm{NAA} / \mathrm{Cr}$ and $\mathrm{Cho} /$ $\mathrm{Cr}$ were found in the LGB and striate regions (76). This indicated that there was an ongoing neurodegenerative process in glaucoma patients. Guo et al. detected the changes of metabolites in patients with early POAG using $1 \mathrm{H}-\mathrm{MRS}$ and found that the average $\mathrm{Glx} / \mathrm{Cr}$ ratio in the primary visual cortex of patients with early POAG was significantly increased, while the average myo-inositol (Ins)/ $\mathrm{Cr}$ ratio was significantly decreased. These findings show that it is helpful to explore the cortical plasticity of the primary visual cortex in patients with early POAG (81).

In animal experiments, Chan et al. conducted a similar study in a rat model with high IOP. At 6 weeks after the rats begun to exhibit high IOP, a low $\mathrm{Cho/Cr}$ ratio was also found in the visual cortex. However, no significant differences were found in other metabolites, including NAA, glutamine, and glutamate (82).

A study of aqueous humor metabolomics based on MRS showed that the typical areas of betaine and taurine in the glaucoma group were higher, which may be related to the neuroprotective mechanism, and the content of typical areas of glutamate was also higher, which may indicate neuronal damage and oxidative stress (77). These findings support the theory of apoptosis, in which glutamate is one of the main factors that leads to neurotoxicity. Similar changes were found in aqueous humor samples from glaucoma patients using gas chromatography and time of flight mass spectrometry (83).

There are some limitations to MRS. Quantification of a single metabolite is usually not feasible due to the presence of a large number of detectable metabolites. Therefore, although they have high diagnostic value, these methods make it more difficult to connect the obtained data with potential metabolic pathways.

\section{MTI}

The MTI method is a selective tissue signal suppression technique in MRI. It is based on the magnetization transfer effect and quantified by magnetization transfer ratio (MTR) (Figure 6). The MTR reflects the protons bound to macromolecules in different tissues, which reveals the degree of proton exchange between free water and macromolecules. It primarily measures the degree of myelination, axon integrity, and macromolecules on the cell membrane of the central nervous system and provides pathological information $(85,86)$.

The magnetization transfer (MT) effect of the brain arises from biological macromolecules in the neuronal cell membrane and organelle membranes, such as cholesterol, galactose cerebroside, and phospholipid in a myelin sheath. With MTR, the ability of these macromolecules to exchange magnetization with surrounding water molecules in the brain is measured (87). The MTI technique has been shown to be very sensitive and able to detect normal morphological changes of the brain. It can be used in optic nerve research to provide quantitative information on tissue integrity, myelin sheath, and axon content (88). The MTR can be measured in different segments of the entire optic nerve, which provides useful information on the progressive nature of demyelination and remyelination over time. The decrease of MTR occurs due to axonal injury caused by demyelination and Waller degeneration, and the increase of MTR may indicate remyelination (89).

Recently, Grochowski et al. proposed a silent-MT sequence using adiabatic spectrum inversion recovery fat suppression pulses on a $7 \mathrm{~T}$ scanner. This silent-MT sequence is silent during operation. The delay between the radio frequency pulse and the signal sampling period is very

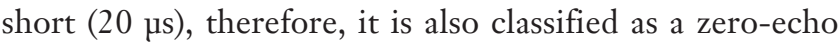
time sequence. Silent-MT imaging has a good signal-tonoise ratio and strong contrast of the optic nerve, good fat suppression, and no motion artifacts. The image generated by this sequence can reveal the whole optic nerve (90). 

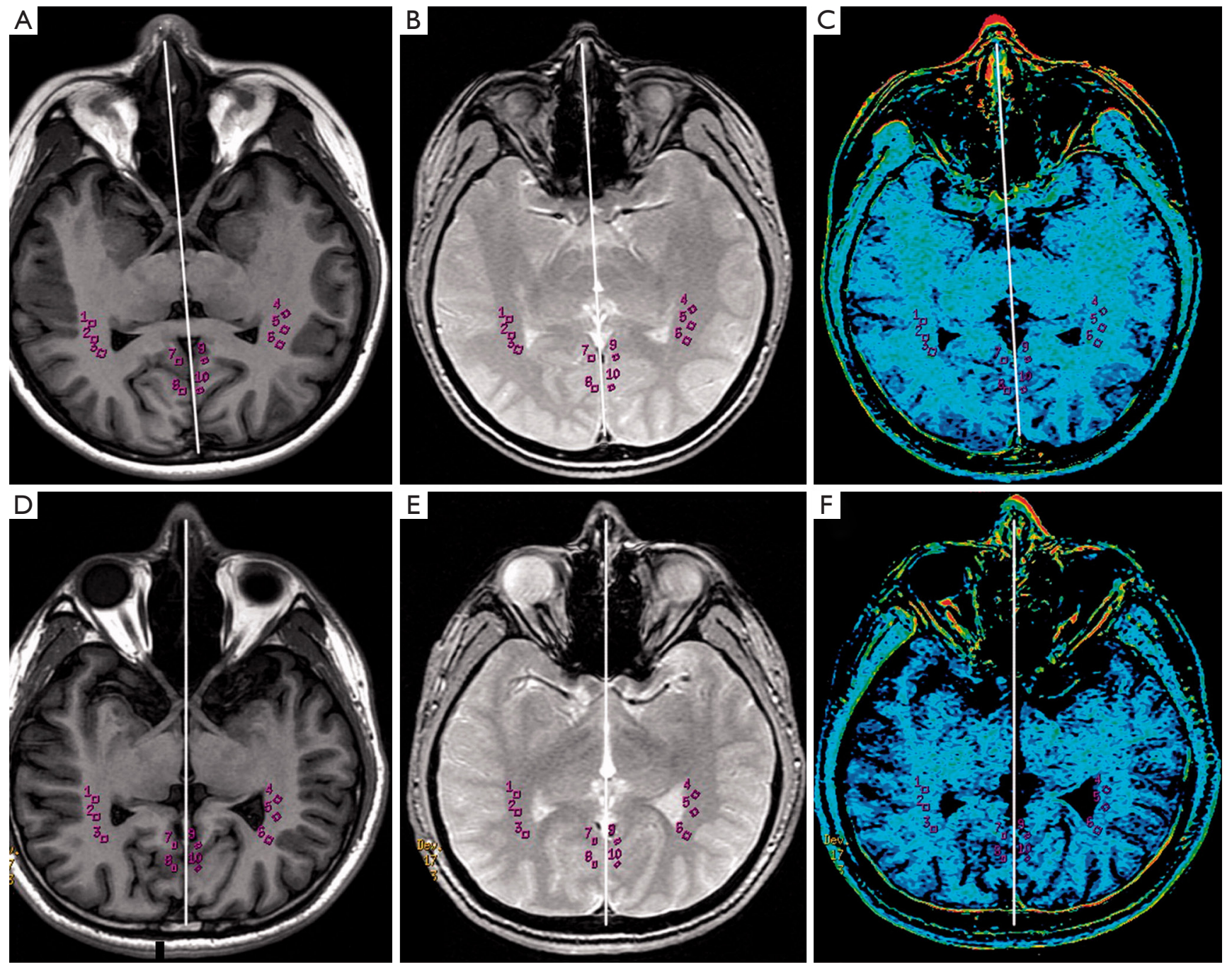

Figure 6 MTI. (A,D) Axial T1-FLAIR images. (B,E) Saturated images acquired with the MT saturation pulse. (C,F) MTR pseudo color maps. (A-C) Images of the brain of a healthy 24-year-old woman. The MTR of her geniculocalcarine and striate area are 35.212 and 32.907, respectively. (D-F) Images of the brain of a 28-year-old female glaucoma patient. The MTR of her geniculocalcarine and striate area are 34.194 and 30.333, respectively. Reprinted with permission from Zhang et al. (84). MTI, magnetization transfer imaging; MTR, magnetization transfer ratio

Few studies have analyzed glaucoma patients with MTI. Kitsos et al. evaluated the brain and optic nerve pathways of 26 patients with POAG and found that the MTR of the optic chiasm, geniculocalcarine, and the visual cortex decreased significantly compared with the control group. They believe that the axonal degeneration and demyelination of the optic nerve pathway and visual cortex may be the reason for the low MTR (91). Zhang et al. studied the geniculocalcarine and striatal region of patients with primary glaucoma by using the region of interest (ROI) analysis of MTR. They found that the
MTR of bilateral geniculocalcarine and striatal region in patients with glaucoma decreased, suggesting that there is geniculocalcarine demyelination and degeneration of striatal region in primary glaucoma. In addition to the direct effect of retrograde axonal degeneration, anterograde transsynaptic changes secondary to the RGCs axon transaction in the optic nerve are a possible mechanism for the decrease of MTR in the striate region (84).

In addition, since MTI measures the MT between free water and water bound to macromolecules through chemical exchange and/or dipole-dipole interaction, it can 
show the changes in macromolecular structure and content. Therefore, MTI can be used to more specifically detect how to organize the microstructure of collagen fibers, and how to reshape the extracellular matrix (92). Ho et al. used freshly prepared amniotic eyes to evaluate the tissue magnetic resonance relaxation in response to dynamic IOP changes. Furthermore, they used MTI to detect the animal model of glaucoma and non-destructively distinguish different eye tissues with and without biomechanical or biochemical regulation. They found that the MTR of the sclera, cornea, optic nerve, and the lens cortex in the glaucoma group was higher than that of the anterior chamber and the vitreous body (93). These studies show that determining the dynamic, biomechanical, and biochemical characteristics of ocular tissue is helpful to evaluate the pathophysiological mechanism of the corneal scleral shell and the efficacy of corneal scleral therapy in various ophthalmic diseases.

Despite its usefulness, MTI has limitations. It cannot identify the different types and stages of glaucoma, which may affect the results of the examination. A larger sample size of homogeneous samples should be carried out, and the clinical indicators of glaucoma, such as IOP and VF defect, should be associated with the changes of MTR to draw more convincing conclusions from studies using MTI.

\section{Discussion}

Glaucoma is a group of diseases characterized by atrophy and depression of the ONH, VF defects, and visual loss. Pathological elevated IOP and insufficient blood supply to the optic nerve are the primary risk factors for developing glaucoma. Primary glaucoma can be divided into 2 patterns according to the state of the anterior chamber angle when IOP increases-PACG and POAG. However, some patients do not have elevated IOP, but there are typical pathological changes of POAG, that is, NTG. The cortical involvement patterns of POAG and PACG are different, which may be related to the different pathogenesis of the 2 glaucoma patterns (36). Although the reasons for the differences between PACG and POAG are not clear, many studies have shown that they have different pathological mechanisms. Anatomically, PACG is characterized by outflow channel obstruction caused by closure of the anterior chamber angle, while POAG is caused by trabecular meshwork damage (94). The thickening of the anterior choroid causes closure of the anterior chamber angle in eyes with PACG, but this is the pathological change caused by the increased IOP in POAG (95). Compared with POAG, the level of pro-inflammatory cytokines in intraocular fluid is lower in PACG (96). There are also differences in the biological processes such as those at the molecular level and complex genetic heterogeneity, among others (97). The different pathological mechanisms of the 2 kinds of glaucoma result in their MRI manifestations being different.

In recent years, with the continuous development of MRI technology, the application of increasingly advanced imaging technology in the field of glaucoma is growing rapidly. Emerging techniques include: fMRI to estimate the synchronization of different regions in the cortical anatomy through BOLD acquisition; MRI quantitative morphometry, DWI, DTI, and DKI to provide quantitative results; MRS to provide different physiological and chemical information by detecting and quantifying biochemical compounds or metabolites in brain tissue; and MTI to quantify pathological information by detecting MTR. These techniques enable us to observe brain lesions of glaucoma from different angles (Table 1).

The introduction of advanced neuroimaging technology may promote research of the whole visual pathway. It may also make the knowledge of pathophysiology more accurate, enable earlier diagnosis of glaucoma than is possible with the current VF, and open a new field in the early detection of glaucoma and the evaluation of the therapeutic effect of new neuroprotective strategies.

However, there are still some limitations in the application of these advanced MRI techniques in the field of glaucoma. First, all MRI studies in the field of glaucoma have been cross-sectional designs and were thus unable to describe the causal relationship of interaction between ocular abnormalities and brain manifestations in glaucoma. Therefore, we cannot assert that glaucoma leads to the reduction of the optic nerve pathway, LGN, or the visual cortex based only on the findings of MRI techniques. The brain lesions associated with the severity of glaucoma remain an area requiring further study involving longitudinal evaluation to compare MRI results with the progression of glaucoma. However, the studies presented in this review have shown that there may be a correlation between glaucoma and brain abnormalities. Second, most of the previous studies have included a small sample size, and there is also a lack of data on the repeatability within or between observers. There are differences in the type, frequency, size, brightness, and color of stimuli in different studies. Therefore, cortical activity will be affected by different stimuli, which will lead to different or even conflicting research conclusions. However, it 
Table 1 Different types of advanced MRI and the main findings in each method

\begin{tabular}{|c|c|c|c|}
\hline Type of MRI & $\begin{array}{l}\text { Type of } \\
\text { glaucoma }\end{array}$ & Authors & Main findings \\
\hline $\begin{array}{l}\text { MRI quantitative } \\
\text { morphometry }\end{array}$ & POAG & Wang et al. (21) & $\begin{array}{l}\text { POAG affects both vision-related structures and non-visual cortical regions. Moreover, } \\
\text { alterations of the brain's visual structures reflect the clinical severity of glaucoma }\end{array}$ \\
\hline $\begin{array}{l}\text { MRI quantitative } \\
\text { morphometry }\end{array}$ & Glaucoma & Wang et al. (20) & $\begin{array}{l}\text { High-tension glaucoma patients experienced morphological reduction in the visual and } \\
\text { nonvisual areas throughout the entire brain. Elevated intraocular pressure may } \\
\text { contribute to the reduction of cortical thickness in certain areas during the progression } \\
\text { of the disease }\end{array}$ \\
\hline $\begin{array}{l}\text { MRI quantitative } \\
\text { morphometry }\end{array}$ & Glaucoma & $\begin{array}{l}\text { Gracitelli et al. } \\
\text { (14) }\end{array}$ & $\begin{array}{l}\text { Glaucoma patients had a decreased occipital pole surface area in both hemispheres } \\
\text { that independently correlated with functional and structural ocular parameters }\end{array}$ \\
\hline $\begin{array}{l}\text { MRI quantitative } \\
\text { morphometry }\end{array}$ & POAG & $\begin{array}{l}\text { Hernowo et al. } \\
\text { (19) }\end{array}$ & $\begin{array}{l}\text { POAG adversely affects structures along the full visual pathway, from the optic nerve } \\
\text { to the optic radiation. Moreover, MRI in combination with automated morphometry can } \\
\text { be used to aid the detection and assessment of glaucomatous damage in the brain }\end{array}$ \\
\hline fMRI & POAG & Dai et al. (32) & $\begin{array}{l}\text { Changes in functional connectivity of the visual cortex are found in patients with } \\
\text { POAG. These include alterations in connectivity between the visual cortex and } \\
\text { associated visual areas along with disrupted connectivity between the primary and } \\
\text { higher visual areas }\end{array}$ \\
\hline fMRI & PACG & Huang et al. (38) & $\begin{array}{l}\text { PACG mainly involved in the dysfunction in the frontal lobe, which may reflect the } \\
\text { underlying pathologic mechanism of PACG }\end{array}$ \\
\hline fMRI & PACG & Jiang et al. (39) & $\begin{array}{l}\text { PACG group showed abnormal spontaneous neural activities in the visual cortices, } \\
\text { sensorimotor cortices, frontal lobe, frontoparietal network, and default mode network } \\
\text { at two frequency bands, which might indicate impaired vision and cognition and } \\
\text { emotion function in PACG individuals }\end{array}$ \\
\hline fMRI & Glaucoma & $\begin{array}{l}\text { Minosse et al. } \\
(25)\end{array}$ & $\begin{array}{l}\text { The differences in local network measurements highlight cerebral reorganization of } \\
\text { brain networks in glaucoma patients, supporting the interpretation that glaucoma is } \\
\text { a central nervous system disease, and is likely part of the heterogeneous group of } \\
\text { recently described disconnection syndromes }\end{array}$ \\
\hline fMRI & NVG & Peng et al. (41) & $\begin{array}{l}\text { The patients with NVG exhibited anomalous spontaneous activity in different brain } \\
\text { regions; these finding should establish the foundation for a more comprehensive } \\
\text { understanding of the pathological mechanisms of NVG. Furthermore, these abnormal } \\
\text { variations in specific brain regions can be considered possible clinical indices of NVG }\end{array}$ \\
\hline fMRI & PACG & Wang et al. (37) & $\begin{array}{l}\text { PACG exhibited abnormal spontaneous neural activity and connectivity in several brain } \\
\text { regions mainly associated with visual and visual-related functions }\end{array}$ \\
\hline fMRI & NVG & Wu et al. (42) & $\begin{array}{l}\text { NVG involves aberrant FC in the V1 in different brain areas, including the visual-related } \\
\text { and cognitive-related regions }\end{array}$ \\
\hline
\end{tabular}

Table 1 (continued) 
Table 1 (continued)

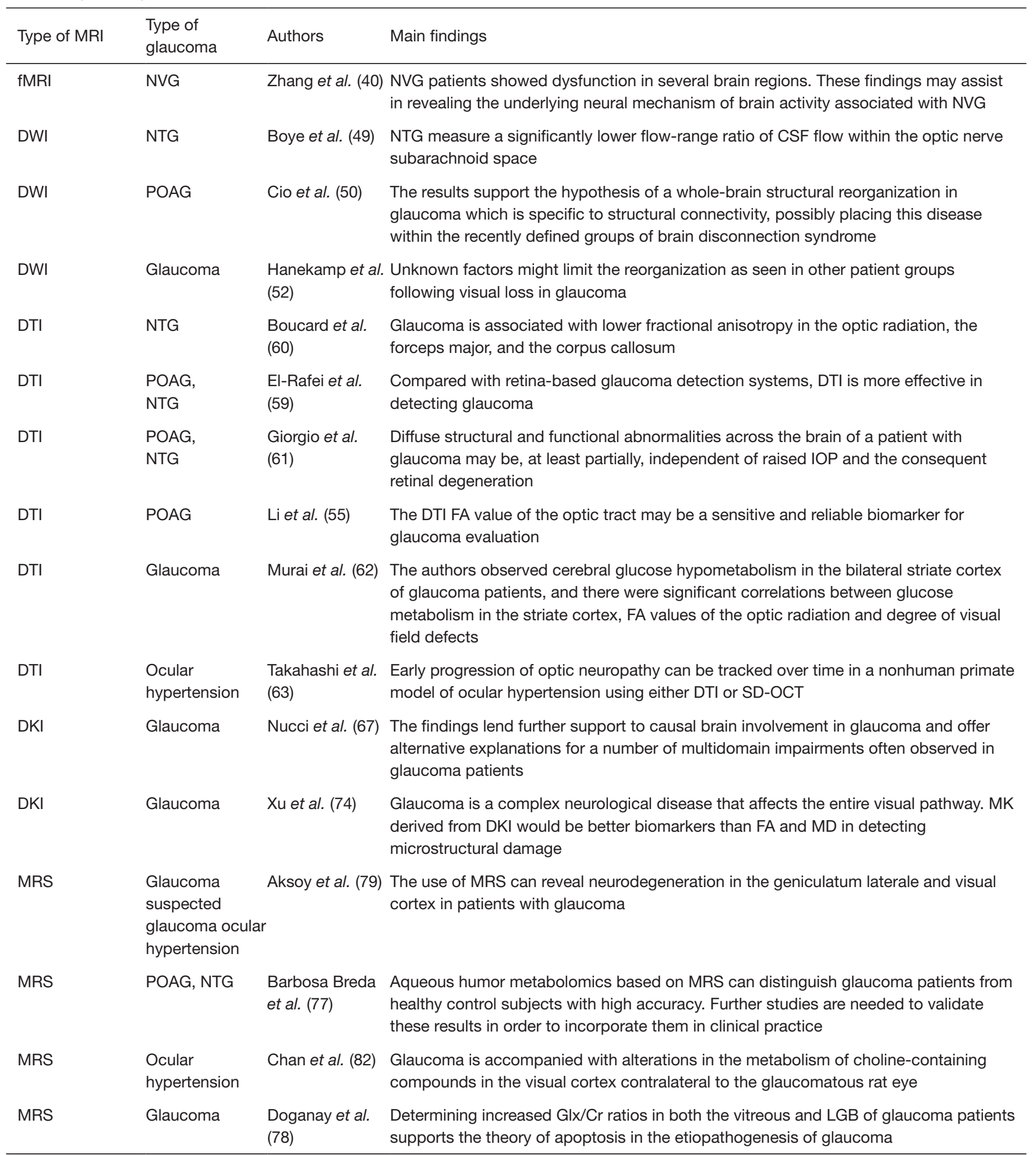

Table 1 (continued) 
Table 1 (continued)

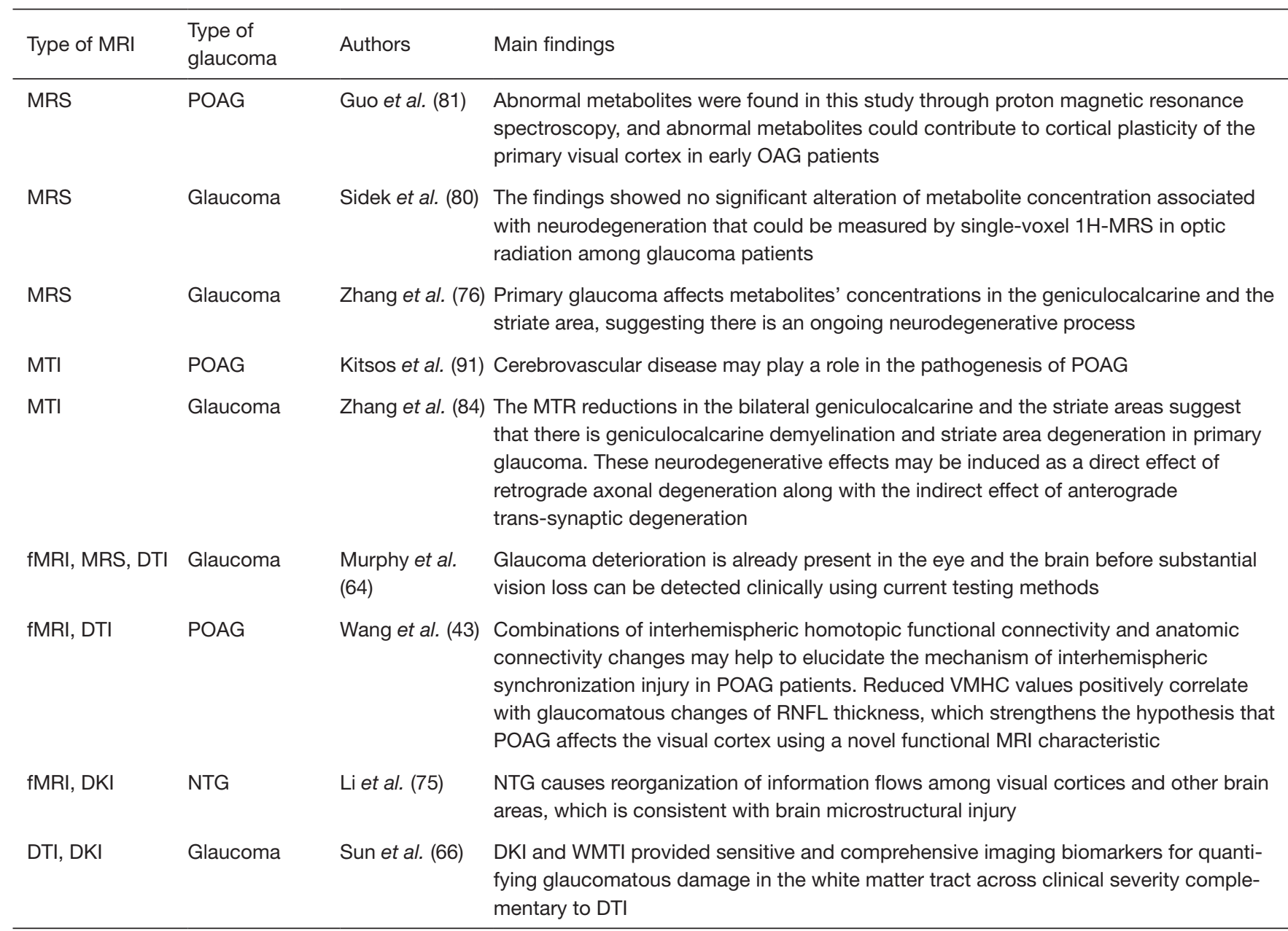

MRI, magnetic resonance imaging; POAG, primary open angle glaucoma; fMRI, functional MRI; PACG, primary angle closure glaucoma; DMN, default mode network; NTG, normal tension glaucoma; NVG, neovascular glaucoma; FC, functional connection; DWI, diffusion-weighted imaging; CSF, cerebrospinal fluid; DTI, diffusion tensor imaging; IOP, intraocular pressure; FA, fractional anisotropy; SD-OCT, spectral domain optical coherence tomography; DKI, diffusion kurtosis imaging; MK, mean kurtosis; MD, mean diffusion coefficient; MRS, magnetic resonance spectroscopy; Glx, glutamate-glutamine; Cr, creatine; LGB, lateral geniculate body; OAG, open angle glaucoma; $1 \mathrm{H}-\mathrm{MRS}$, monomer proton MRS; MTI, magnetization transfer imaging; MTR, magnetization transfer ratio; VMHC, voxel-mirrored homotopic connectivity; RNFL, retinal nerve fiber layer; WMTI, white matter tract integrity.

should be emphasized that MRI is not a routine glaucoma examination. The MRI examination for glaucoma patients is particularly challenging. Due to its complexity and cost, it is also a time-consuming examination. Even in global medical centers, modern $3 \mathrm{~T}$ or $7 \mathrm{~T}$ MRI equipment cannot be widely used. Third, aging and systemic or local treatment may affect the results of brain analysis. We note that most published MRI studies have included homogeneous samples in their methods, but some have a large range of age, different degrees of glaucoma injury (stage), or different treatment methods, which may affect the final results. In addition, most of these patients use beta-blockers, prostaglandin analogs, and/or carbonic anhydrase inhibitors. A recent study using Rs-fMRI to evaluate glaucoma patients shows that the subtle effects of these drugs on internal brain dynamics have not been determined (98). Finally, although different studies have reported significant associations in the correlation analysis between intraocular structures and brain abnormalities, the correlation analysis involving ions and/or the LGN is unclear. Therefore, it is necessary to 
further clarify the complexity of neural networks related to visual pathways, in terms of sample characteristics, data acquisition protocols, and applied statistical models.

New research is ongoing to better elucidate glaucoma damage throughout the visual pathway. In addition, some researchers are committed to incorporating complementary high-definition technology into our daily practice to overcome some of our current limitations in dealing with glaucoma, including difficulties in disease diagnosis and monitoring. Some new insights and studies are as follows. First, longitudinal design research in glaucoma populations tries to understand the speed of damage of the visual pathway (such as the change rate of brain structure) and works to identify the early signals of glaucoma. Second, at present, the evaluation methods of glaucoma using standard automatic VF measurement, optic disc photos, and OCT cannot be replaced by MRI. However, we should not only pay attention to the eye injury of patients with glaucoma, but also place focus on the related brain injury. Therefore, MRI should also be one of the important tools for glaucoma evaluation. In future, the detection of cerebral neurodegeneration described in these studies may open new fields in the early detection of diseases and the evaluation of therapeutic effects of new neuroprotective strategies. Third, most of the previously cited studies were based on 3T MRI equipment, but now we have 7T MRI, which has higher resolution and a more powerful function of acquisition and scanning. This reduces the impact of artifacts, and the actual damage can be better identified and quantified. Further studies should use 7T MRI to evaluate glaucoma patients. Finally, glaucoma is increasingly being considered a neurodegenerative disease that affects the retina and central visual pathway. Some researchers even believe that it is essentially a disease of the central nervous system. Many studies have observed degenerative changes in the LGN, LGB, and visual cortex in patients with glaucoma; however, the nature of glaucoma remains unknown. Determining whether glaucoma is an anterograde disease originating from RGC degeneration, a retrograde disease originating from the visual cortex or the LGN, or the combined result of the 2 mechanisms, that is, whether the changes in the brain occur before, at the same time, or after the ocular lesions, has been controversial (99). Moving forward, it is necessary to conduct large-scale research to clarify the principle of brain participation in glaucoma and determine the common pathological mechanism that leads to neurodegeneration in glaucoma and other diseases.

Increasing evidence shows that glaucoma affects the whole visual pathway. The application of advanced MRI methods increases our in-depth understanding of cortical reorganization, neural plasticity, and reconstruction of visual pathways in patients with glaucoma. The results of the structural, functional, and metabolic analysis showed that the injury of RGCs could induce the cross synaptic degeneration of the LGN and the visual cortex. Almost all changes of RGCs in the optic nerve were accompanied by changes in a posterior visual pathway or the nervous system. These changes are not limited to the primary visual cortex, but also involve other brain regions that are not directly involved in visual information processing. In addition, the connection between the primary and higher visual cortex, and between the visual cortex and related regions, is destroyed. A better understanding of the changes in the brain as a result of glaucoma can help us better understand the nature and progress of vision loss. New insights into changes within the brain may enable us to analyze the course of glaucoma progression and stimulate new strategies for neuroprotective therapy of glaucoma in the future.

\section{Acknowledgments}

Funding: This work was supported by grants from Natural Science Foundation of Xinjiang Province (2020D01A122 to C Wan); Natural Science Foundation of Liaoning Province (2020-MS-01 to C Wan). And the work was supported by Key Laboratory of Intelligent Computing in Medical Image, Ministry of Education.

\section{Footnote}

Reporting Checklist: The authors have completed the Narrative Review reporting checklist. Available at https:// dx.doi.org/10.21037/qims-21-790

Conflicts of Interest: Both authors have completed the ICMJE uniform disclosure form (available at https://dx.doi. org/10.21037/qims-21-790). Both authors have no conflicts of interest to declare.

Ethical Statement: The authors are accountable for all aspects of the work in ensuring that questions related to the accuracy or integrity of any part of the work are appropriately investigated and resolved.

Open Access Statement: This is an Open Access article distributed in accordance with the Creative Commons 
Attribution-NonCommercial-NoDerivs 4.0 International License (CC BY-NC-ND 4.0), which permits the noncommercial replication and distribution of the article with the strict proviso that no changes or edits are made and the original work is properly cited (including links to both the formal publication through the relevant DOI and the license). See: https://creativecommons.org/licenses/by-nc-nd/4.0/.

\section{References}

1. Youngblood H, Hauser MA, Liu Y. Update on the genetics of primary open-angle glaucoma. Exp Eye Res 2019;188:107795.

2. Stein JD, Khawaja AP, Weizer JS. Glaucoma in AdultsScreening, Diagnosis, and Management: A Review. JAMA 2021;325:164-74.

3. Salam AA, Khalil T, Akram MU, Jameel A, Basit I. Automated detection of glaucoma using structural and non structural features. Springerplus 2016;5:1519.

4. Senger C, Moreto R, Watanabe SES, Matos AG, Paula JS. Electrophysiology in Glaucoma. J Glaucoma 2020;29:147-53.

5. Jonas JB, Aung T, Bourne RR, Bron AM, Ritch R, PandaJonas S. Glaucoma. Lancet 2017;390:2183-93.

6. Schuster AK, Erb C, Hoffmann EM, Dietlein T, Pfeiffer N. The Diagnosis and Treatment of Glaucoma. Dtsch Arztebl Int 2020;117:225-34.

7. McMonnies CW. Glaucoma history and risk factors. J Optom 2017;10:71-8.

8. Torres LA, Hatanaka M. Correlating Structural and Functional Damage in Glaucoma. J Glaucoma 2019;28:1079-85.

9. Sun Y, Huang W, Li F, Li H, Wang L, Huang Y, Zhang X. Subcortical visual pathway may be a new way for early diagnosis of glaucoma. Med Hypotheses 2019;123:47-9.

10. Wang Q, Qu X, Chen W, Wang H, Huang C, Li T, Wang N, Xian J. Altered coupling of cerebral blood flow and functional connectivity strength in visual and higher order cognitive cortices in primary open angle glaucoma. J Cereb Blood Flow Metab 2021;41:901-13.

11. You M, Rong R, Zeng Z, Xia X, Ji D. Transneuronal Degeneration in the Brain During Glaucoma. Front Aging Neurosci 2021;13:643685.

12. Artero-Castro A, Rodriguez-Jimenez FJ, Jendelova P, VanderWall KB, Meyer JS, Erceg S. Glaucoma as a Neurodegenerative Disease Caused by Intrinsic Vulnerability Factors. Prog Neurobiol 2020;193:101817. 13. Siesky B, Wentz SM, Januleviciene I, Kim DH, Burgett
KM, Verticchio Vercellin AC, Rowe LW, Eckert GJ, Harris A. Baseline structural characteristics of the optic nerve head and retinal nerve fiber layer are associated with progressive visual field loss in patients with open-angle glaucoma. PLoS One 2020;15:e0236819.

14. Gracitelli CPB, Duque-Chica GL, Sanches LG, Moura AL, Nagy BV, Teixeira SH, Amaro E Jr, Ventura DF, Paranhos A Jr. Structural Analysis of Glaucoma Brain and its Association With Ocular Parameters. J Glaucoma 2020;29:393-400.

15. Mastropasqua R, Agnifili L, Mattei PA, Caulo M, Fasanella V, Navarra R, Mastropasqua L, Marchini G. Advanced Morphological and Functional Magnetic Resonance Techniques in Glaucoma. Biomed Res Int 2015;2015:160454.

16. Campbell JS, Pike GB. Potential and limitations of diffusion MRI tractography for the study of language. Brain Lang 2014;131:65-73.

17. Hoch MJ, Bruno MT, Shepherd TM. Advanced MRI of the Optic Nerve. J Neuroophthalmol 2017;37:187-96.

18. Yu F, Duong T, Tantiwongkosi B. Advanced MR Imaging of the Visual Pathway. Neuroimaging Clin N Am 2015;25:383-93.

19. Hernowo AT, Boucard CC, Jansonius NM, Hooymans JM, Cornelissen FW. Automated morphometry of the visual pathway in primary open-angle glaucoma. Invest Ophthalmol Vis Sci 2011;52:2758-66.

20. Wang Y, Wang X, Zhou J, Qiu J, Yan T, Xie Y, Li L, Lu W. Brain morphological alterations of cerebral cortex and subcortical nuclei in high-tension glaucoma brain and its associations with intraocular pressure. Neuroradiology 2020;62:495-502.

21. Wang J, Li T, Sabel BA, Chen Z, Wen H, Li J, Xie X, Yang D, Chen W, Wang N, Xian J, He H. Structural brain alterations in primary open angle glaucoma: a $3 \mathrm{~T}$ MRI study. Sci Rep 2016;6:18969.

22. Furlanetto RL, Teixeira SH, Gracitelli CPB, Lottenberg CL, Emori F, Michelan M, Amaro E Jr, Paranhos A Jr. Structural and functional analyses of the optic nerve and lateral geniculate nucleus in glaucoma. PLoS One 2018;13:e194038.

23. Murphy MC, Nau AC, Fisher C, Kim SG, Schuman JS, Chan KC. Top-down influence on the visual cortex of the blind during sensory substitution. Neuroimage 2016;125:932-40.

24. Sims JR, Chen AM, Sun Z, Deng W, Colwell NA, Colbert MK, Zhu J, Sainulabdeen A, Faiq MA, Bang JW, Chan KC. Role of Structural, Metabolic, and Functional MRI 
in Monitoring Visual System Impairment and Recovery. J Magn Reson Imaging 2021;54:1706-29.

25. Minosse S, Floris R, Nucci C, Toschi N, Garaci F, Martucci A, Lanzafame S, Di Giuliano F, Picchi E, Cesareo M, Mancino R, Guerrisi M. Disruption of brain network organization in primary open angle glaucoma. Annu Int Conf IEEE Eng Med Biol Soc 2019;2019:4338-41.

26. Martucci A, Cesareo M, Toschi N, Garaci F, Bagetta G, Nucci C. Brain networks reorganization and functional disability in glaucoma. Prog Brain Res 2020;257:65-76.

27. Qi CX, Huang X, Shen Y. Altered Intrinsic Brain Activities in Patients with Diabetic Retinopathy Using Amplitude of Low-frequency Fluctuation: A Resting-state fMRI Study. Diabetes Metab Syndr Obes 2020;13:2833-42.

28. Su T, Yuan Q, Liao XL, Shi WQ, Zhou XZ, Lin Q, Min YL, Li B, Jiang N, Shao Y. Altered intrinsic functional connectivity of the primary visual cortex in patients with retinal vein occlusion: a resting-state fMRI study. Quant Imaging Med Surg 2020;10:958-69.

29. Tang A, Chen T, Zhang J, Gong Q, Liu L. Abnormal Spontaneous Brain Activity in Patients With Anisometropic Amblyopia Using Resting-State Functional Magnetic Resonance Imaging. J Pediatr Ophthalmol Strabismus 2017;54:303-10.

30. Wang Y, Lu W, Yan T, Zhou J, Xie Y, Yuan J, Liu G, Teng Y, Han W, Chen D, Qiu J. Functional MRI reveals effects of high intraocular pressure on central nervous system in high-tension glaucoma patients. Acta Ophthalmol 2019;97:e341-8.

31. Zhou W, Muir ER, Nagi KS, Chalfin S, Rodriguez P, Duong TQ. Retinotopic fMRI Reveals Visual Dysfunction and Functional Reorganization in the Visual Cortex of Mild to Moderate Glaucoma Patients. J Glaucoma 2017;26:430-7.

32. Dai H, Morelli JN, Ai F, Yin D, Hu C, Xu D, Li Y. Resting-state functional MRI: functional connectivity analysis of the visual cortex in primary open-angle glaucoma patients. Hum Brain Mapp 2013;34:2455-63.

33. Li T, Liu Z, Li J, Liu Z, Tang Z, Xie X, Yang D, Wang N, Tian J, Xian J. Altered amplitude of lowfrequency fluctuation in primary open-angle glaucoma: a resting-state FMRI study. Invest Ophthalmol Vis Sci 2014;56:322-9.

34. Li HL, Chou XM, Liang Y, Pan T, Zhou Q, Pei CG, Jiang J, Li B, Shao Y. Use of rsfMRI-fALFF for the detection of changes in brain activity in patients with normal-tension glaucoma. Acta Radiol 2021;62:414-22.

35. Qing G, Zhang S, Wang B, Wang N. Functional MRI signal changes in primary visual cortex corresponding to the central normal visual field of patients with primary open-angle glaucoma. Invest Ophthalmol Vis Sci 2010;51:4627-34.

36. Chen W, Zhang L, Xu YG, Zhu K, Luo M. Primary angle-closure glaucomas disturb regional spontaneous brain activity in the visual pathway: an fMRI study. Neuropsychiatr Dis Treat 2017;13:1409-17.

37. Wang R, Tang Z, Liu T, Sun X, Wu L, Xiao Z. Altered spontaneous neuronal activity and functional connectivity pattern in primary angle-closure glaucoma: a resting-state fMRI study. Neurol Sci 2021;42:243-51.

38. Huang X, Zhong YL, Zeng XJ, Zhou F, Liu XH, Hu PH, Pei CG, Shao Y, Dai XJ. Disturbed spontaneous brain activity pattern in patients with primary angle-closure glaucoma using amplitude of low-frequency fluctuation: a fMRI study. Neuropsychiatr Dis Treat 2015;11:1877-83.

39. Jiang F, Yu C, Zuo MJ, Zhang C, Wang Y, Zhou FQ, Zeng XJ. Frequency-dependent neural activity in primary angle-closure glaucoma. Neuropsychiatr Dis Treat 2019;15:271-82.

40. Zhang YQ, Peng MY, Wu SN, Yu CY, Chen SY, Tan SW, Shao Y, Zhou Q. Fractional amplitude of low-frequency fluctuation in patients with neovascular glaucoma: a resting-state functional magnetic resonance imaging study. Quant Imaging Med Surg 2021;11:2138-50.

41. Peng ZY, Liu YX, Li B, Ge QM, Liang RB, Li QY, Shi WQ, Yu YJ, Shao Y. Altered spontaneous brain activity patterns in patients with neovascular glaucoma using amplitude of low-frequency fluctuations: A functional magnetic resonance imaging study. Brain Behav 2021;11:e02018.

42. Wu YY, Wang SF, Zhu PW, Yuan Q, Shi WQ, Lin Q, Li B, Min YL, Zhou Q, Shao Y. Altered Intrinsic Functional Connectivity of the Primary Visual Cortex in Patients with Neovascular Glaucoma: A Resting-State Functional Magnetic Resonance Imaging Study. Neuropsychiatr Dis Treat 2020;16:25-33.

43. Wang Q, Chen W, Wang H, Zhang X, Qu X, Wang Y, Li T, Wang N, Xian J. Reduced Functional and Anatomic Interhemispheric Homotopic Connectivity in Primary Open-Angle Glaucoma: A Combined Resting State-fMRI and DTI Study. Invest Ophthalmol Vis Sci 2018;59:1861-8.

44. Nakajo Y, Zhao Q, Enmi JI, Iida H, Takahashi JC, Kataoka H, Yamato K, Yanamoto H. Early Detection of Cerebral Infarction After Focal Ischemia Using a New MRI Indicator. Mol Neurobiol 2019;56:658-70.

45. Haykal S, Jansonius NM, Cornelissen FW. Investigating 
changes in axonal density and morphology of glaucomatous optic nerves using fixel-based analysis. Eur J Radiol 2020;133:109356.

46. Tian Y, Wang J, Li M, Lou X, Tang J, Xu Q, Zhang Y, Wei S, Ma L. Comparison of field-of-view optimized and constrained undistorted single-shot diffusion-weighted imaging and conventional diffusion-weighted imaging of optic nerve and chiasma at 3T. Neuroradiology 2018;60:903-12.

47. Seeger A, Schulze M, Schuettauf F, Ernemann U, Hauser TK. Advanced diffusion-weighted imaging in patients with optic neuritis deficit - value of reduced field of view DWI and readout-segmented DWI. Neuroradiol J 2018;31:126-32.

48. Klingebiel M, Ullrich T, Quentin M, Bonekamp D, Aissa J, Mally D, Arsov C, Albers P, Antoch G, Schimmöller L. Advanced diffusion weighted imaging of the prostate: Comparison of readout-segmented multi-shot, paralleltransmit and single-shot echo-planar imaging. Eur J Radiol 2020;130:109161.

49. Boye D, Montali M, Miller NR, Pircher A, Gruber P, Killer HE, Remonda L, Berberat J. Flow dynamics of cerebrospinal fluid between the intracranial cavity and the subarachnoid space of the optic nerve measured with a diffusion magnetic resonance imaging sequence in patients with normal tension glaucoma. Clin Exp Ophthalmol 2018;46:511-8.

50. Cio FD, Garaci F, Minosse S, Passamonti L, Martucci A, Lanzafame S, Giuliano FD, Picchi E, Mancino R, Guerrisi M, Nucci C, Floris R, Toschi N. Disruption of structural brain networks in Primary Open Angle Glaucoma. Annu Int Conf IEEE Eng Med Biol Soc 2020;2020:1705-8.

51. Di Ciò F, Garaci F, Minosse S, Passamonti L, Martucci A, Lanzafame S, Di Giuliano F, Picchi E, Cesareo M, Guerrisi MG, Floris R, Nucci C, Toschi N. Reorganization of the structural connectome in primary open angle Glaucoma. Neuroimage Clin 2020;28:102419.

52. Hanekamp S, Ćurčić-Blake B, Caron B, McPherson B, Timmer A, Prins D, Boucard CC, Yoshida M, Ida M, Hunt D, Jansonius NM, Pestilli F, Cornelissen FW. White matter alterations in glaucoma and monocular blindness differ outside the visual system. Sci Rep 2021;11:6866.

53. Schmidt MA, Knott M, Heidemann R, Michelson G, Kober T, Dörfler A, Engelhorn T. Investigation of lateral geniculate nucleus volume and diffusion tensor imaging in patients with normal tension glaucoma using 7 tesla magnetic resonance imaging. PLoS One 2018;13:e0198830.
54. Lilja Y, Nilsson DT. Strengths and limitations of tractography methods to identify the optic radiation for epilepsy surgery. Quant Imaging Med Surg 2015;5:288-99.

55. Li M, Ke M, Song Y, Mu K, Zhang H, Chen Z. Diagnostic utility of central damage determination in glaucoma by magnetic resonance imaging: An observational study. Exp Ther Med 2019;17:1891-5.

56. Gao L, Huang M, Luo X, Song T, Wang X, Wang Z, Zhang W, Chen J, Lei H. Microstructure abnormity in the optic nerve of type 1 diabetic rats revealed by diffusion tensor imaging study. Magn Reson Imaging 2020;71:105-14.

57. Wang L, Fan K, Zhang Y, Chen Y, Tian Q, Shi D. Quantitative assessment of optic nerve in patients with Leber's hereditary optic neuropathy using reduced field-of-view diffusion tensor imaging. Eur J Radiol 2017;93:24-9.

58. Engelhorn T, A Schmidt M, Dörfler A, Michelson G. Diffusion tensor imaging of the visual pathway in glaucomatous optic nerve atrophy. Ophthalmologe 2017;114:906-21.

59. El-Rafei A, Engelhorn T, Wärntges S, Dörfler A, Hornegger J, Michelson G. Glaucoma classification based on visual pathway analysis using diffusion tensor imaging. Magn Reson Imaging 2013;31:1081-91.

60. Boucard CC, Hanekamp S, Ćurčić-Blake B, Ida M, Yoshida M, Cornelissen FW. Neurodegeneration beyond the primary visual pathways in a population with a high incidence of normal-pressure glaucoma. Ophthalmic Physiol Opt 2016;36:344-53.

61. Giorgio A, Zhang J, Costantino F, De Stefano N, Frezzotti P. Diffuse brain damage in normal tension glaucoma. Hum Brain Mapp 2018;39:532-41.

62. Murai H, Suzuki Y, Kiyosawa M, Tokumaru AM, Ishiwata $\mathrm{K}$, Ishii K. Cerebral glucose metabolism in the striate cortex positively correlates with fractional anisotropy values of the optic radiation in patients with glaucoma. Clin Exp Ophthalmol 2015;43:711-9.

63. Takahashi N, Matsunaga N, Natsume T, Kitazawa C, Itani Y, Hama A, Hayashi I, Shimazawa M, Hara H, Takamatsu H. A longitudinal comparison in cynomolgus macaques of the effect of brimonidine on optic nerve neuropathy using diffusion tensor imaging magnetic resonance imaging and spectral domain optical coherence tomography. Heliyon 2021;7:e06701.

64. Murphy MC, Conner IP, Teng CY, Lawrence JD, Safiullah Z, Wang B, Bilonick RA, Kim SG, Wollstein G, Schuman JS, Chan KC. Retinal Structures and Visual Cortex Activity are Impaired Prior to Clinical Vision Loss in 
Glaucoma. Sci Rep 2016;6:31464.

65. You Y, Joseph C, Wang C, Gupta V, Liu S, Yiannikas C, Chua BE, Chitranshi N, Shen T, Dheer Y, Invernizzi A, Borotkanics R, Barnett M, Graham SL, Klistorner A. Demyelination precedes axonal loss in the transneuronal spread of human neurodegenerative disease. Brain 2019;142:426-42.

66. Sun Z, Parra C, Bang JW, Fieremans E, Wollstein G, Schuman JS, Chan KC. Diffusion Kurtosis Imaging Reveals Optic Tract Damage That Correlates with Clinical Severity in Glaucoma. Annu Int Conf IEEE Eng Med Biol Soc 2020;2020:1746-9.

67. Nucci C, Garaci F, Altobelli S, Di Ciò F, Martucci A, Aiello F, Lanzafame S, Di Giuliano F, Picchi E, Minosse S, Cesareo M, Guerrisi MG, Floris R, Passamonti L, Toschi N. Diffusional Kurtosis Imaging of White Matter Degeneration in Glaucoma. J Clin Med 2020;9:3122.

68. Lanzafame S, Giannelli M, Garaci F, Floris R, Duggento A, Guerrisi M, Toschi N. Differences in Gaussian diffusion tensor imaging and non-Gaussian diffusion kurtosis imaging model-based estimates of diffusion tensor invariants in the human brain. Med Phys 2016;43:2464.

69. Jensen JH, Helpern JA. MRI quantification of nonGaussian water diffusion by kurtosis analysis. NMR Biomed 2010;23:698-710.

70. Li HQ, Yin B, Quan C, Geng DY, Yu H, Bao YF, Liu J, Li YX. Evaluation of patients with relapsing-remitting multiple sclerosis using tract-based spatial statistics analysis: diffusion kurtosis imaging. BMC Neurol 2018;18:108.

71. Winston GP. The potential role of novel diffusion imaging techniques in the understanding and treatment of epilepsy. Quant Imaging Med Surg 2015;5:279-87.

72. De Santis S, Bastiani M, Droby A, Kolber P, Zipp F, Pracht E, Stoecker T, Groppa S, Roebroeck A. Characterizing Microstructural Tissue Properties in Multiple Sclerosis with Diffusion MRI at $7 \mathrm{~T}$ and $3 \mathrm{~T}$ : The Impact of the Experimental Design. Neuroscience 2019;403:17-26.

73. Thaler C, Kyselyova AA, Faizy TD, Nawka MT, Jespersen S, Hansen B, Stellmann JP, Heesen C, Stürner KH, Stark M, Fiehler J, Bester M, Gellißen S. Heterogeneity of multiple sclerosis lesions in fast diffusional kurtosis imaging. PLoS One 2021;16:e0245844.

74. Xu ZF, Sun JS, Zhang XH, Feng YY, Pan AZ, Gao MY, Zhao H. Microstructural visual pathway abnormalities in patients with primary glaucoma: $3 \mathrm{~T}$ diffusion kurtosis imaging study. Clin Radiol 2018;73:591.e9-591.e15.

75. Li T, Qu X, Chen W, Wang Q, Wang H, Wang Y, Huang
C, Zhang X, Wang N, Xian J. Altered information flow and microstructure abnormalities of visual cortex in normal-tension glaucoma: Evidence from resting-state fMRI and DKI. Brain Res 2020;1741:146874.

76. Zhang Y, Chen X, Wen G, Wu G, Zhang X. Proton magnetic resonance spectroscopy ((1)H-MRS) reveals geniculocalcarine and striate area degeneration in primary glaucoma. PLoS One 2013;8:e73197.

77. Barbosa Breda J, Croitor Sava A, Himmelreich U, Somers A, Matthys C, Rocha Sousa A, Vandewalle E, Stalmans I. Metabolomic profiling of aqueous humor from glaucoma patients - The metabolomics in surgical ophthalmological patients (MISO) study. Exp Eye Res 2020;201:108268.

78. Doganay S, Cankaya C, Alkan A. Evaluation of corpus geniculatum laterale and vitreous fluid by magnetic resonance spectroscopy in patients with glaucoma; a preliminary study. Eye (Lond) 2012;26:1044-51.

79. Aksoy DÖ, Umurhan Akkan JC, Alkan A, Aralaşmak A, Otçu Temur H, Yurtsever İ. Magnetic Resonance Spectroscopy Features of the Visual Pathways in Patients with Glaucoma. Clin Neuroradiol 2019;29:615-21.

80. Sidek S, Ramli N, Rahmat K, Ramli NM, Abdulrahman F, Kuo TL. In vivo proton magnetic resonance spectroscopy (1H-MRS) evaluation of the metabolite concentration of optic radiation in primary open angle glaucoma. Eur Radiol 2016;26:4404-12.

81. Guo L, Wang R, Tang Z, Sun X, Wu L, Wang J, Zhong Y, Xiao Z, Zhang Z. Metabolic Alterations Within the Primary Visual Cortex in Early Open-angle Glaucoma Patients: A Proton Magnetic Resonance Spectroscopy Study. J Glaucoma 2018;27:1046-51.

82. Chan KC, So KF, Wu EX. Proton magnetic resonance spectroscopy revealed choline reduction in the visual cortex in an experimental model of chronic glaucoma. Exp Eye Res 2009;88:65-70.

83. Chen X, Chen Y, Wang L, Sun X. Metabolomics of the aqueous humor in patients with primary congenital glaucoma. Mol Vis 2019;25:489-501.

84. Zhang Y, Liang W, Wu G, Zhang X, Wen G. Magnetization transfer imaging reveals geniculocalcarine and striate area degeneration in primary glaucoma: a preliminary study. Acta Radiol Open 2016;5:2058460116666876.

85. Maleki S, Chye Y, Zhang X, Parkes L, Chamberlain SR, Fontenelle LF, Braganza L, Youssef G, Lorenzetti V, Harrison BJ, Yücel M, Suo C. Neural correlates of symptom severity in obsessive-compulsive disorder using magnetization transfer and diffusion tensor imaging. 
Psychiatry Res Neuroimaging 2020;298:111046.

86. Tambasco N, Nigro P, Romoli M, Simoni S, Parnetti L, Calabresi P. Magnetization transfer MRI in dementia disorders, Huntington's disease and parkinsonism. J Neurol Sci 2015;353:1-8.

87. Ercan E, Varma G, Mädler B, Dimitrov IE, Pinho MC, Xi Y, Wagner BC, Davenport EM, Maldjian JA, Alsop DC, Lenkinski RE, Vinogradov E. Microstructural correlates of $3 \mathrm{D}$ steady-state inhomogeneous magnetization transfer (ihMT) in the human brain white matter assessed by myelin water imaging and diffusion tensor imaging. Magn Reson Med 2018;80:2402-14.

88. Lebel C, Deoni S. The development of brain white matter microstructure. Neuroimage 2018;182:207-18.

89. van Gelderen P, Duyn JH. Background suppressed magnetization transfer MRI. Magn Reson Med 2020;83:883-91.

90. Grochowski C, Symms M, Jonak K, Krukow P, Wood T, Ljungberg E, Enseñat J, Nowomiejska K, Rejdak R, Maciejewski R, Barker GJ. The Evaluation of Optic Nerves Using 7 Tesla "Silent" Zero Echo Time Imaging in Patients with Leber's Hereditary Optic Neuropathy with or without Idebenone Treatment. J Clin Med 2020;9:1112.

91. Kitsos G, Zikou AK, Bagli E, Kosta P, Argyropoulou MI. Conventional MRI and magnetisation transfer imaging of the brain and optic pathway in primary open-angle glaucoma. Br J Radiol 2009;82:896-900.

92. Magat J, Fouillet A, Constantin M, Haliot K, Naulin J, El Hamrani D, Benoist D, Charron S, Walton R, Bernus O, Quesson B. 3D magnetization transfer (MT) for the visualization of cardiac free-running Purkinje fibers: an ex vivo proof of concept. MAGMA 2021;34:605-18.

93. Ho LC, Sigal IA, Jan NJ, Yang X, van der Merwe Y,

Cite this article as: Kang L, Wan C. Application of advanced magnetic resonance imaging in glaucoma: a narrative review. Quant Imaging Med Surg 2022;12(3):2106-2128. doi: 10.21037/ qims-21-790
Yu Y, Chau Y, Leung CK, Conner IP, Jin T, Wu EX, Kim SG, Wollstein G, Schuman JS, Chan KC. Noninvasive MRI Assessments of Tissue Microstructures and Macromolecules in the Eye upon Biomechanical or Biochemical Modulation. Sci Rep 2016;6:32080.

94. Kondkar AA. Updates on Genes and Genetic Mechanisms Implicated in Primary Angle-Closure Glaucoma. Appl Clin Genet 2021;14:89-112.

95. Gao K, Li F, Li Y, Li X, Huang W, Chen S, Liu Y, Aung T, Zhang X. Anterior Choroidal Thickness Increased in Primary Open-Angle Glaucoma and Primary AngleClosure Disease Eyes Evidenced by Ultrasound Biomicroscopy and SS-OCT. Invest Ophthalmol Vis Sci 2018;59:1270-7.

96. Chua J, Vania M, Cheung CM, Ang M, Chee SP, Yang H, Li J, Wong TT. Expression profile of inflammatory cytokines in aqueous from glaucomatous eyes. Mol Vis 2012;18:431-8.

97. Seet LF, Narayanaswamy A, Finger SN, Htoon HM, Nongpiur ME, Toh LZ, Ho H, Perera SA, Wong TT. Distinct iris gene expression profiles of primary angle closure glaucoma and primary open angle glaucoma and their interaction with ocular biometric parameters. Clin Exp Ophthalmol 2016;44:684-92.

98. Minosse S, Garaci F, Martucci A, Lanzafame S, Di Giuliano F, Picchi E, Cesareo M, Mancino R, Guerrisi M, Pistolese CA, Floris R, Nucci C, Toschi N. Primary Open Angle Glaucoma Is Associated With Functional Brain Network Reorganization. Front Neurol 2019;10:1134.

99. Prins D, Hanekamp S, Cornelissen FW. Structural brain MRI studies in eye diseases: are they clinically relevant? A review of current findings. Acta Ophthalmol 2016;94:113-21. 